\title{
Mass Functions of Supermassive Black Holes across Cosmic Time
}

\author{
Brandon C. Kelly1, ${ }^{1}$ and Andrea Merloni' \\ ${ }^{1}$ High Energy Astrophysics Division, Harvard-Smithsonian Center for Astrophysics, 60 Garden Street, Cambridge, MA 02138, USA \\ ${ }^{2}$ Department of Physics, Broida Hall, University of California, Santa Barbara, Santa Barbara, CA 93106, USA \\ ${ }^{3}$ Max-Planck-Institut für Extraterrestrische Physik, Giessenbachstraße 1, 85748 Garching, Germany \\ Correspondence should be addressed to Brandon C. Kelly, bckelly@cfa.harvard.edu
}

Received 10 September 2011; Accepted 1 December 2011

Academic Editor: Francesca Civano

Copyright (๑) 2012 B. C. Kelly and A. Merloni. This is an open access article distributed under the Creative Commons Attribution License, which permits unrestricted use, distribution, and reproduction in any medium, provided the original work is properly cited.

\begin{abstract}
The black hole mass function of supermassive black holes describes the evolution of the distribution of black hole mass. It is one of the primary empirical tools available for mapping the growth of supermassive black holes and for constraining theoretical models of their evolution. In this paper, we discuss methods for estimating the black hole mass function, including their advantages and disadvantages. We also review the results of using these methods for estimating the mass function of both active and inactive black holes. In addition, we review current theoretical models for the growth of supermassive black holes that predict the black hole mass function. We conclude with a discussion of directions for future research which will lead to improvement in both empirical and theoretical determinations of the mass function of supermassive black holes.
\end{abstract}

\section{Introduction}

Understanding how and when supermassive black holes (SMBHs) grow is currently of central importance in extragalactic astronomy. A significant amount of empirical work has established correlations between SMBH mass and host galaxy spheroidal properties, such as luminosity [1-3], stellar velocity dispersion (the $M_{\mathrm{BH}}-\sigma_{*}$ relationship, e.g., [4-6]), concentration or Sersic index [7,8], bulge mass [9-11], and binding energy $[12,13]$. These scaling relationships imply that the evolution of spheroidal galaxies and the growth of SMBHs are intricately tied together. The currently favored mechanism for linking the growth of SMBHs and their hosts is black hole feedback, whereby black holes grow by accreting gas in the so-called "active" phases, possibly fueled by a major merger of two gas-rich galaxies, until feedback energy from the SMBH expels gas and shuts off the accretion process [1417]. Alternatively, it has been suggested that the origin of the scaling relationships does not necessarily require $\mathrm{SMBH}$ feedback but emerges from the stochastic nature of the hierarchical assembly of black hole and stellar mass through galaxy mergers $[18,19]$.

Feedback-driven "self-regulated" growth of black holes has been able to reproduce the local $M_{\mathrm{BH}}-\sigma_{*}$ relationship in smoothed particle hydrodynamics simulations [20-22]. Moreover, AGN feedback has also been invoked as a means of quenching the growth of the most massive galaxies [23, 24]. There have been numerous models linking $\mathrm{SMBH}$ growth, the quasar phase, and galaxy evolution [25-33]. While feedback is likely important for regulating the growth of SMBHs and galaxies, the fueling mechanisms that contribute to growing the $\mathrm{SMBH}$ are likely diverse. Major mergers of gasrich galaxies may fuel quasars at high redshift and grow the most massive SMBHs. However, major mergers alone do not appear to be sufficient to reproduce the number of X-ray faint AGN [34], and accretion of ambient gas via internal galactic processes $[35,36]$ may fuel these fainter, lower $M_{\mathrm{BH}}$ AGN at lower $z$. This is supported by the fact that many AGN are observed to live in late-type galaxies out to $z \approx 1[37,38]$, and the X-ray luminosity function of AGN hosted by latetype galaxies suggests that fueling by minor interactions or internal instabilities represents a nonnegligible contribution to the accretion history of the universe [39].

The black hole mass function (BHMF) provides a complete census of the mass of SMBHs and their evolution. Because of this, the BHMF is one of the primary empirical tools available for investigating the growth of SMBHs, and for constraining theoretical models for the growth of the SMBH 
population. Because SMBHs and galaxies are thought to be linked in their evolution, the BHMF provides insight into the fueling mechanisms that dominate black hole growth and therefore into the role of feedback in the evolution of the host galaxy. The BHMF is also an important tool in planning future surveys, as it provides an estimate of the distribution of SMBH mass expected for the survey. This in turn is important because mass is a fundamental quantity of the black hole and therefore is an important observational quantity for empirical studies of black hole accretion physics [40-45]. Of course, further improvement to our understanding of black hole accretion physics will further improve our modeling and understanding of black hole accretion and feedback, which in turn will improve our understanding of black-hole-galaxy coevolution. Therefore, the BHMF is an important empirical quantity for SMBH studies.

In this paper, we discuss the current status of BHMF estimation and theoretical modeling. In Section 2, we discuss the nontrivial task of estimating the BHMF. In Section 3, we discuss current estimates of the local SMBH BHMF. In Section 4, we discuss BHMF estimates derived by combining the local BHMF with the AGN luminosity function via a continuity equation. In Section 5, we discuss BHMFs estimated for AGN only. In Section 6, we review theoretical models for $\mathrm{SMBH}$ growth that predict the SMBH BHMF. Finally, in Section 7, we discuss directions for future improvements to the empirical and theoretical studies of the BHMF. We note that unlike, say, the luminosity function, the division between "observational" and "theoretical" studies is not as clear for the BHMF, as some amount of modeling is necessary in order to estimate the BHMF from strictly observational quantities. We have attempted to divide the studies according to whether the BHMF is constrained empirically, as in, say, a formal statistical fitting procedure, or if it is predicted from a theoretical model for $\mathrm{SMBH}$ growth. In reality, the line between theoretical and empirical studies is blurry and some procedures which we have considered to be empirical may be thought of as theoretical.

\section{Estimating the Black Hole Mass Function}

The black hole mass function, denoted as $\phi\left(M_{\mathrm{BH}}, z\right) d M_{\mathrm{BH}}$, is the number of sources per comoving volume $V(z)$ with black hole masses in the range $M_{\mathrm{BH}}, M_{\mathrm{BH}}+d M_{\mathrm{BH}}$. The black hole mass function is related to the joint probability distribution of $M_{\mathrm{BH}}$ and $z, p\left(M_{\mathrm{BH}}, z\right)$, as

$$
\phi\left(M_{\mathrm{BH}}, z\right)=N\left(\frac{d V}{d z}\right)^{-1} p\left(M_{\mathrm{BH}}, z\right) .
$$

The normalization of the BHMF is $N$, the total number of SMBHs in the observable universe, and is given by the integral of $\phi$ over $M_{\mathrm{BH}}$ and $V(z)$.

\subsection{Complications with Estimating Black Hole Mass Functions.} Similar to luminosity function estimation, the BHMF may be estimated from astronomical surveys. However, while there are many well-established methods for estimating luminosity functions, there are two complications that make BHMF estimation a more difficult problem [46]. The first is the issue of incompleteness. Surveys are typically constructed by finding the set of objects of interest containing a SMBH that satisfy a flux criteria, for example, all objects brighter than some flux limit. Surveys are not constructed by selecting on mass. Because there is a distribution of luminosities at a given $\mathrm{SMBH}$ mass, whether it is the luminosity of the host galaxy or of the AGN, some SMBHs will scatter above the flux limit and some below. This creates a selection function which is less sensitive to $M_{\mathrm{BH}}$, and it is possible that a survey may be incomplete in all mass bins.

The second complication is the large uncertainty in $\mathrm{SMBH}$ mass among mass estimators. Currently, it is not possible to obtain reliable mass estimators for large numbers of SMBHs through dynamical and modeling of the stellar or gaseous components, and thus scaling relationships are employed. Masses may be estimated using scaling relationships between $M_{\mathrm{BH}}$ and the properties of the host galaxy bulge or the luminosity and the width of the broad emission lines for AGN [47, 48]. It has also recently been suggested that the X-ray variability properties of AGN may also provide another scaling relationship for estimating $M_{\mathrm{BH}}[45,49,50]$, but further work is needed for developing this. While these scaling relationships enable one to estimate $M_{\mathrm{BH}}$ for large numbers of SMBHs, they also contain a significant intrinsic statistical scatter. Gültekin et al. [51] find that for early-type galaxies there is an intrinsic scatter in $M_{\mathrm{BH}}$ of $0.31 \pm 0.06 \mathrm{dex}$ and $0.38 \pm 0.09$ dex at fixed host galaxy bulge dispersion and luminosity, respectively; the amplitude of the scatter is larger for late-type galaxies. For AGN with broad emission lines, Vestergaard and Peterson [48] estimate the scatter in $M_{\mathrm{BH}}$ at fixed luminosity and line width to be $\sim 0.4 \mathrm{dex}$, depending on which emission line is used.

The statistical uncertainty in the mass estimates can have a significant effect on the inferred BHMF. The distribution of the mass estimates is the convolution of the intrinsic BHMF with the error distribution in the mass estimates. In general, it is typically assumed that the error in the mass estimates is independent of the actual value of $M_{\mathrm{BH}}$. This is not the case for $M_{\mathrm{BH}}$ estimated through dynamical modeling; however, independence between $M_{\mathrm{BH}}$ and its error is likely to be a good approximation for $M_{\mathrm{BH}}$ estimated using scaling relationships. Because scaling relationships are the only feasible manner to estimate $M_{\mathrm{BH}}$ for a large sample of SMBHs, which is necessary for any estimate of the BHMF, we will assume that $M_{\mathrm{BH}}$ and its error are independent. Under the assumption of independence between the estimated $M_{\mathrm{BH}}$ and its error, the BHMF that would be inferred directly from the distribution of the mass estimates is broader than the intrinsic BHMF and is thus biased. Figure 1 illustrates this effect, where an intrinsic mass function is compared with the distribution of an unbiased mass estimator having a statistical uncertainty of $0.3,0.4$, and $0.5 \mathrm{dex}$, respectively. As can be seen, the distribution of mass estimates is significantly different from the intrinsic mass function. In particular, the distribution of the mass estimates falls off more slowly with increasing $M_{\mathrm{BH}}$, and overpredicts the number of SMBHs at the high $M_{\mathrm{BH}}$ end of the mass function. The bias is worse when the dispersion in the scatter in the mass estimates becomes larger. 


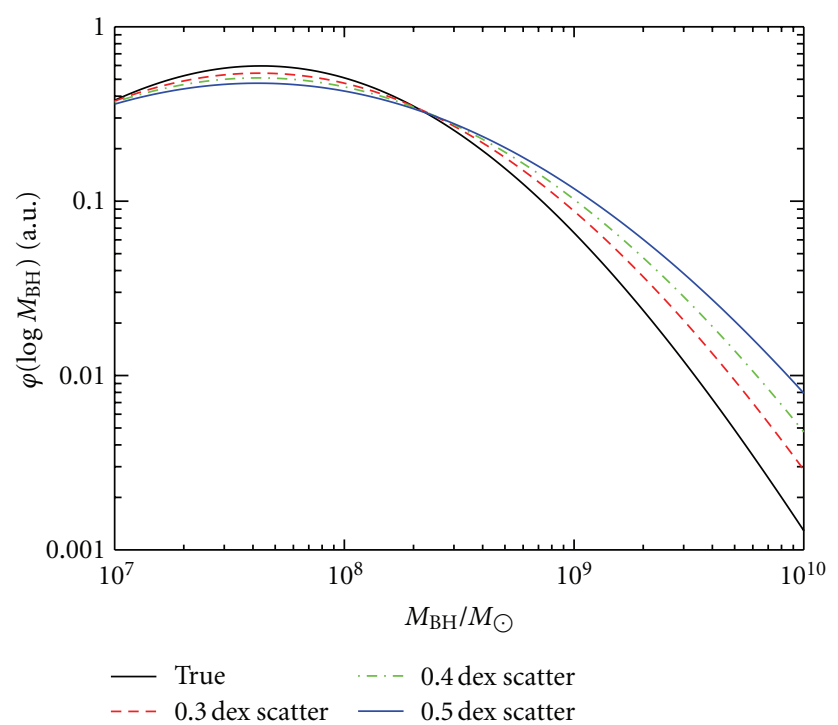

FIGURE 1: Illustration of the bias in the estimated BHMF derived from mass estimates. Shown is the true mass function (thick solid black line) for a simulated sample, and the mass function derived from the mass estimateswhen the statistical error in the mass estimates is $0.3 \mathrm{dex}$ (red dashed line), $0.4 \mathrm{dex}$ (green dot-dashed line), and $0.5 \mathrm{dex}$ (solid blue thin line). The mass function estimated from the mass estimates is biased, especially at the high-MBH end and for large statistical error.

2.2. Methodology for Estimating the Black Hole Mass Function. In order to estimate the SMBH mass function in an unbiased manner, it is necessary to match the mass function with the observed distribution of the mass estimates and any additional observational quantities that the selection function (The selection function is the probability of including a source in one's sample as a function of its measured quantities) depends on. The basic idea is to start with an assumed mass function. Then, calculate the distribution of mass estimates implied by this mass function. In addition, calculate the distribution of observational quantities that one's sample is selected on, say, flux, that is implied by the assumed mass function. This step allows one to correct for incompleteness but requires an additional assumption about how to relate the mass function to the quantity that one's sample is selected on. Finally, impose the selection function for the sample and compare the predicted observed distributions of mass estimates and any other observables (e.g., flux) with the actual distributions. If they are not consistent, then the data rule out the assumed mass function and relationship between $M_{\mathrm{BH}}$ and the observable quantities.

We can make the above procedure more quantitative by deriving the likelihood function for the $\mathrm{SMBH}$ mass function. Kelly et al. [46] derived the likelihood function for the mass function when using masses estimated from AGN broad emission lines. They used this likelihood function for developing a Bayesian approach to estimating the $\mathrm{SMBH}$ mass function. Although their method was limited to broadline mass estimates, it is straightforward to generalize their formalism for any generic mass estimator. Denote the black hole mass estimate as $\widehat{M}_{\mathrm{BH}}$. In addition, denote as $X$ the set of observables that one uses to select one's sample. In the majority of cases, this will be flux at one or more wavelengths. Then, the likelihood function for the BHMF based on a sample of $n$ SMBHs is

$$
\begin{aligned}
p\left(\widehat{\mathbf{M}}_{\mathrm{BH}}, \mathbf{X}, \mathbf{z} \mid \theta, \psi, N\right) & \\
\propto C_{n}^{N}[s(\theta, \psi)]^{N-n} \prod_{i=1}^{n} \int_{0}^{\infty} & p\left(\widehat{M}_{\mathrm{BH}, i} \mid M_{\mathrm{BH}, i}, z_{i}, X_{i}\right) \\
& \times p\left(X_{i} \mid M_{\mathrm{BH}, i}, z_{i}, \psi\right) \\
& \times p\left(M_{\mathrm{BH}, i}, z_{i} \mid \theta\right) d M_{\mathrm{BH}, i},
\end{aligned}
$$

where the BHMF is related to $N$ and the probability distribution of $M_{\mathrm{BH}}$ and $z$ via (1). Here, $C_{n}^{N}$ is the binomial coefficient, $\theta$ denotes the parameters for the BHMF, $\psi$ denotes the parameters for the distribution in $X$ at fixed $M_{\mathrm{BH}}$ and $z$, and $s(\theta, \psi)$ is the probability of including a SMBH in one's sample as a function of $\theta$ and $\psi$. Here, we have assumed that the distribution in the mass estimates at fixed $M_{\mathrm{BH}}, z$, and $X$, $p\left(\widehat{M}_{\mathrm{BH}} \mid M_{\mathrm{BH}}, z, X\right)$ is known, although one could include additional free parameters for this as well. The binomial coefficient arises from the fact that the number of objects included in one's survey follows a binomial distribution (Often in the luminosity function literature the likelihood is assumed to be a Poisson distribution. A poisson distribution is an approximation to the binomial distribution when $N \rightarrow \infty$ and $s(\theta, \psi) \rightarrow 0$, so (2) converges to the Poisson distribution when $n \ll N$. See [52] for further details.) with $N$ "trials" and probability of success $s(\theta, \psi)$. The probability of including a SMBH in one's survey as a function of the BHMF, $s(\theta, \psi)$, is calculated from the survey selection function $s(X, z)$ as

$$
\begin{aligned}
s(\theta, \psi)=\int_{X_{\min }}^{X_{\max }} s(X, z)\left[\iint_{0}^{\infty} p\left(X \mid M_{\mathrm{BH}}, z, \psi\right)\right. \\
\left.\quad \times p\left(M_{\mathrm{BH}}, z \mid \theta\right) d M_{\mathrm{BH}} d z\right] d X .
\end{aligned}
$$

It is up to the researcher to choose the particular parametric form for the SMBH mass function, the distribution in the mass estimates at fixed $M_{\mathrm{BH}}, z$, and $X$, and the distribution of the observable that the sample is selected on (e.g., flux) at fixed $M_{\mathrm{BH}}$ and $z$. Typical choices are log-normal distributions, Schechter functions, and mixtures of log-normal distributions. Once one has done this, one can use (2) to compute a maximum-likelihood estimate for the BHMF or perform Bayesian inference.

An alternative form of estimating the BHMF can be used when the mass estimates are derived from an observational quantity, $Y$, and the intrinsic distribution of $Y$ is known. This is commonly used to estimate the local mass function using host-galaxy scaling relationships [53]. In this case, the mass function is

$$
\phi\left(M_{\mathrm{BH}}\right)=\int_{Y_{\min }}^{Y_{\max }} p\left(M_{\mathrm{BH}} \mid Y\right) \phi(Y) d Y,
$$


where $\phi(Y)$ is the comoving number density of SMBHs as a function of the quantity $Y$. When both $p\left(M_{\mathrm{BH}} \mid Y\right)$ and $\phi(Y)$ are known, then the BHMF follows directly from (4). As an example, if the mass function is derived from the scaling between $M_{\mathrm{BH}}$ and host galaxy spheroidal luminosity, $L_{\mathrm{sph}}$, then $Y=L_{\text {sph }}, p\left(M_{\mathrm{BH}} \mid Y\right)$ is the $M_{\mathrm{BH}}-L_{\text {sph }}$ relationship and $\phi(Y)$ is the luminosity function of stellar bulges hosting SMBHs. As with BHMFs determined from a mass estimator, improper treatment of the intrinsic scatter in $M_{\mathrm{BH}}$ at fixed $Y$ will lead to a biased estimate of the BHMF. However, when calculating the BHMF from (4), ignoring the intrinsic scatter results in an estimated BHMF that is too narrow, underpredicting the number of SMBHs at the high-mass end of $\phi\left(M_{\mathrm{BH}}\right)$. This is opposite to the case when one estimates the BHMF directly from mass estimates.

\section{Black Hole Mass Functions Derived from Host Galaxy Scaling Relationships}

The observed scaling between $M_{\mathrm{BH}}$ and the properties of the SMBH host galaxy bulge have motivated several groups to estimate the local BHMF [53-65], with decreasing statistical uncertainties. These estimates of the local BHMF have formed the basis for many studies which have attempted to map black hole growth by comparing with the AGN luminosity function, this is further discussed in Section 4. Typically, the local BHMF is estimated using the local $M_{\mathrm{BH}^{-}}$ $\sigma_{*}$ relationship or the local $M_{\mathrm{BH}}-L_{\text {sph }}$ relationship, combined with the local number density of galaxies as a function of stellar velocity dispersion or bulge luminosity.

The scaling relationships between $M_{\mathrm{BH}}$ and host galaxy properties are only determined for the local universe, and thus most authors have limited their determination of the BHMF based on them to the local BHMF. There are, however, a couple of exceptions. Tamura et al. [66], estimated the BHMF out to $z \approx 1$ assuming that evolution in the $M_{\mathrm{BH}}-L_{\mathrm{sph}}$ relation is driven only by passive evolution in $L_{\text {sph }}$. Shankar et al. [67] (see also [68, 69]) used the local velocity dispersion function of spheroids in combination with their inferred age distributions to estimate the BHMF at $z<6$. In order to do this, they assumed that most of the stars in nearby spheroids formed in a single event and that $\sigma_{*}$ did not change once the spheroid was formed. In addition, Shankar et al. [67] allowed the normalization of the $M_{\mathrm{BH}}-\sigma_{*}$ relationship to evolve, with the degree of evolution being a free parameter. They found evidence for mild evolution in the normalization of the $\mathrm{M}_{\mathrm{BH}^{-}}$ $\sigma_{*}$ relationship.

Evolution in the scaling relationships is currently an area of intense study, with most groups finding evidence that the normalization of the scaling relationships increases towards higher $z$ [70-75], at least for active SMBHs. However, there are still concerns regarding potential biases due to selection effects [76], but see Treu et al. [70] and Bennert et al. [75] for procedures aimed at modeling and correcting for selection. There may also be biases due to extrapolating the AGN mass estimates derived from the broad emission lines to luminous quasars at high $z$ [77]. As such, the uncertainties on the quantitative form of the evolution in the scaling relationships and their scatter are currently large, limiting their use for determining the BHMF outside of the local universe.

When the $M_{\mathrm{BH}}-\sigma_{*}$ relationship is used to estimate the local BHMF, it is common to use the velocity dispersion distribution derived from the SDSS by Sheth et al. [78], with an additional component representing the brightest cluster galaxies [58]. Sheth et al. [78] estimate the velocity dispersion distribution for late-type galaxies by using the Tully-Fisher relation to convert the luminosity function of late-type galaxies to a circular velocity distribution and then set $\sigma=v_{c} / \sqrt{2}$. When the $M_{\mathrm{BH}}-L_{\text {sph }}$ relation is used, it is typical to estimate the distribution of $L_{\text {sph }}$ separately for early- and late-type galaxies by converting their respective luminosity functions to spheroidal luminosity functions using an assumed ratio of bulge luminosity to total luminosity. From this, it has been inferred that the local BHMF is dominated by earlytype galaxies at $M_{\mathrm{BH}} \gtrsim 4 \times 10^{7} M_{\odot}$ [62]. Shankar et al. [64] present a compilation of recently determined local BHMFs based on a variety of methods, scaling relations used, and data sets used. In Figure 2, we show the range of local BHMFs estimated from the $M_{\mathrm{BH}^{-}} \sigma_{*}, M_{\mathrm{BH}^{-}} L_{\text {sph }}$, and $M_{\mathrm{BH}^{-}}$ $M_{\text {star }}$ relationships, as presented in Shankar et al. [64]. In general, estimates of the local mass density of SMBHs span the range $\rho_{\mathrm{BH}}=(3.2-5.4) \times 10^{5} M_{\odot} \mathrm{Mpc}^{-3}$ for $h=0.7$ [64].

While the procedure for estimating the local BHMF is, in theory, straightforward, a number of significant systematics remain. First, there is the observational difficulty that most BHMFs derived from the $M_{\mathrm{BH}}-\sigma_{*}$ relationship are based on SDSS spectra. Unfortunately, the SDSS velocity dispersions are based on a fixed aperture, and thus the size of the aperture relative to the bulge varies with the apparent size of the galaxy and its inclination. In addition, the spectral resolution of SDSS spectra is $\sim 100 \mathrm{kms}^{-1}$, making it difficult to reliably measure $\sigma_{*}$ for SMBHs with $M_{\mathrm{BH}}<10^{7} M_{\odot}$. Another concern is that the local BHMF is derived by assuming that the $M_{\mathrm{BH}}-\sigma_{*}$ or $M_{\mathrm{BH}}-L_{\mathrm{sph}}$ relations are single power laws with a constant scatter in $M_{\mathrm{BH}}$ at fixed $\sigma_{*}$ or $L_{\mathrm{sph}}$. However, recent work has shown these assumptions to be incorrect. For one, the $M_{\mathrm{BH}}-\sigma_{*}$ and $M_{\mathrm{BH}}-L_{\mathrm{sph}}$ relations diverge at the high- $M_{\mathrm{BH}}$ end, which Lauer et al. [58] suggest implies that the $M_{\mathrm{BH}}-\sigma_{*}$ relation is not a single power law. This divergence creates an inconsistency in the BHMFs derived from these two scaling relationships $[58,61]$. Similarly, the $\sigma-L$ relationships for the SDSS and dynamical $M_{\mathrm{BH}} \mathrm{SMBH}$ samples are inconsistent, suggesting a possible selection bias in the estimated BHMFs $[55,79]$. The scatter in the $M_{\mathrm{BH}}-\sigma_{*}$ relation is larger for spirals $[51,80]$, and appears to increase at low $M_{\mathrm{BH}}$ such that most SMBHs lie below the $M_{\mathrm{BH}}-\sigma_{*}$ relation, see (e.g., [81]). Several authors have found differences in the slope and scatter of the scaling relations for pseudobulges [80, 82-84]; however, it is unclear that this result is due to differences in the perceived bulge velocity dispersions for bulges as compared to pseudobulges or due to different scaling relationships. Recently, Kormendy et al. [85] argue that $M_{\mathrm{BH}}$ does not correlate with galaxy disks and only correlates weakly, if at all, with pseudobulges. On other hand, Graham et al. [86] analyzed a larger sample of barred galaxies and concluded that $M_{\mathrm{BH}}$ does correlate with $\sigma_{*}$, even though the $M_{\mathrm{BH}^{-}} \sigma_{*}$ relationship for barred galaxies 


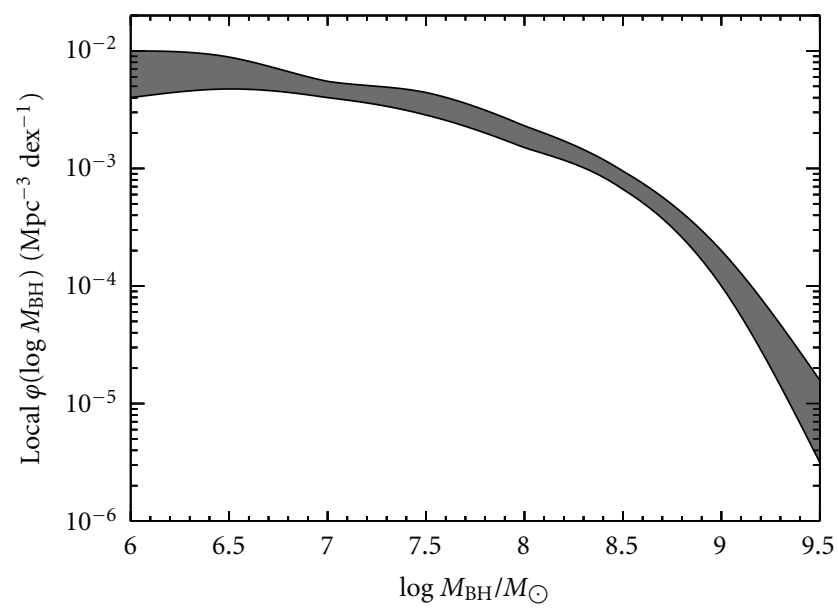

FIGURE 2: Local BHMF. The shaded region defines the spread in estimates obtained using the $M_{\mathrm{BH}}-\sigma_{*}, M_{\mathrm{BH}}-L_{\mathrm{sph}}$, and $M_{\mathrm{BH}}-M_{\text {star }}$ relationships, as compiled by Shankar et al. [64]. Based on this estimate, the local universe is dominated by SMBHs with $M_{\mathrm{BH}}<10^{7} M_{\odot}$.

is offset from that of non barred galaxies. Although there is still much that we do not understand about the $M_{\mathrm{BH}}$ and host galaxy scaling relationship, these recent results suggest that the scaling relationships are not a single power law with constant intrinsic dispersion in $M_{\mathrm{BH}}$, representing a significant source of systematic uncertainty in the estimated local BHMF, especially at the low-mass end.

\section{Black Hole Mass Functions Derived from the Local Mass Function and the AGN Luminosity Function}

By employing the argument of Soltan [87], numerous studies have attempted to estimate the BHMF at a variety of redshifts by comparing the accreted mass distribution implied by the quasar luminosity function with the local BHMF [53, 55, 63, 88-92]. These methods employ a continuity equation describing the evolution of the number density of SMBHs $[93,94]$ :

$$
\frac{\partial \phi_{M}\left(M_{\mathrm{BH}}, t\right)}{\partial t}+\frac{\partial \phi_{M}\left(M_{\mathrm{BH}}, t\right)\left\langle\dot{M}\left(M_{\mathrm{BH}}, t\right)\right\rangle}{\partial M_{\mathrm{BH}}}=n_{\text {merge }}\left(M_{\mathrm{BH}}, t\right) .
$$

Here, $\left\langle\dot{M}\left(M_{\mathrm{BH}}, t\right)\right\rangle$ is the average growth rate of SMBHs as a function of $M_{\mathrm{BH}}$ and cosmic age, $t(z)$, and $n_{\text {merge }}\left(M_{\mathrm{BH}}, t\right)$ is the rate at which the number density of SMBHs changes due to mergers of black holes, or ejections of black holes from their host galaxies due to gravitational recoil. Technically, $n_{\text {merge }}\left(M_{\mathrm{BH}}, t\right)$ can also include a contribution from SMBHs which are created, but this has not been thought to occur over the redshift range in which (5) is typically applied, that is, $z \lesssim 5$. Because the merger rate of black holes is currently unknown, many studies that have employed (5) set $n_{\text {merge }}\left(M_{\mathrm{BH}}, t\right)=0$.

Under the assumption that SMBHs grow during phases of AGN activity, AGN demographics in combination with the local BHMF may be used to compute $\phi_{M}\left(M_{\mathrm{BH}}, t\right)$. This is because the AGN luminosity function maps the accretion history onto SMBHs, and the local BHMF acts as a boundary condition on (5); it is also possible in principle to include the BHMF for AGN, which provides more information. Studies that have used (5) to estimate the BHMF generally fall into two categories: those that assume an AGN lightcurve and those that employ the BHMF of AGN. We discuss each of these separately.

4.1. Methods That Assume an AGN Lightcurve. Most authors employing (5) have assumed a parametric form for $\left\langle\dot{M}\left(M_{\mathrm{BH}}, t\right)\right\rangle$. The accretion rate is related to the bolometric luminosity output of the accretion flow onto the SMBH as $L=\epsilon_{r} \dot{M}_{\mathrm{acc}} c^{2}$, where $\epsilon_{r}$ is the radiative efficiency of the accretion flow, $\dot{M}_{\text {acc }}$ is the accretion rate of matter onto the $\mathrm{SMBH}$, and $c$ is the speed of light. The growth rate of the SMBH is $\dot{M}=\left(1-\epsilon_{r}\right) \dot{M}_{\text {acc }}$, due to the fact that a fraction $\epsilon_{r}$ of accreted mass is radiated away as energy. Making this substitution, the continuity equation becomes

$$
\frac{\partial \phi_{M}\left(M_{\mathrm{BH}}, t\right)}{\partial t}+\frac{1-\epsilon_{r}}{\epsilon_{r} c^{2}} \frac{\partial \phi_{M}\left(M_{\mathrm{BH}}, t\right)\left\langle L\left(M_{\mathrm{BH}}, t\right)\right\rangle}{\partial M_{\mathrm{BH}}}=0,
$$

where we have ignored mergers of SMBHs. Equation (6) shows that it is possible to calculated the BHMF at a time given the local BHMF, an assumed average accretion flow lightcurve as a function of $M_{\mathrm{BH}},\left\langle L\left(M_{\mathrm{BH}}, t\right)\right\rangle$, and an assumed radiative efficiency. Because $\phi_{M}\left(M_{\mathrm{BH}}, z\right)$ and $\left\langle L\left(M_{\mathrm{BH}}, t(z)\right)\right\rangle$ imply a luminosity function, the local BHMF and AGN luminosity function can be used to place constraints on $\epsilon_{r}$ and $\left\langle L\left(M_{\mathrm{BH}}, t\right)\right\rangle$. This means that, in practice, one also has to assume a bolometric correction, which itself likely depends on both black hole mass [95] and $L / L_{\text {Edd }}[96,97]$. In addition, an estimate of $\left\langle L\left(M_{\mathrm{BH}}, t\right)\right\rangle$ also enables one to estimate the lifetime and duty cycle of AGN activity, modulo some luminosity-dependent definition of an AGN; note that the AGN duty cycle defines the fraction of SMBHs that are "active" at a given $M_{\mathrm{BH}}$ and $z$.

A variety of lightcurve models have been used when employing (6) to reconstruct the evolution of the BHMF. The simplest model is that where SMBHs spend a fraction of their time radiating at a constant Eddington ratio and spend the remainder of their time in quiescence. The free parameters in this model are the Eddington ratio, AGN lifetime or duty cycle, and radiative efficiency. This model has been used by [53-55, 57, 64] (technically, [54] assumed that the Eddington ratio was a weakly increasing function of luminosity) to study the build-up of the local black hole mass function, although [64] also considered models where the average accretion rate relative to Eddington falls off toward lower $z$ and higher $M_{\mathrm{BH}}$. Raimundo and Fabian [98] employed a variation on the constant $L / L_{\mathrm{Edd}}$ models, assuming three different populations of AGN with their own Eddington ratio: a population of obscured low $L / L_{\text {Edd }} A G N$, a population of obscured AGN with higher $L / L_{\mathrm{Edd}}$, and a population of unobscured AGN. Yu and $\mathrm{Lu}$ [62] modeled the quasar lightcurve as radiating at the Eddington limit for a period of time, and then transitioning 


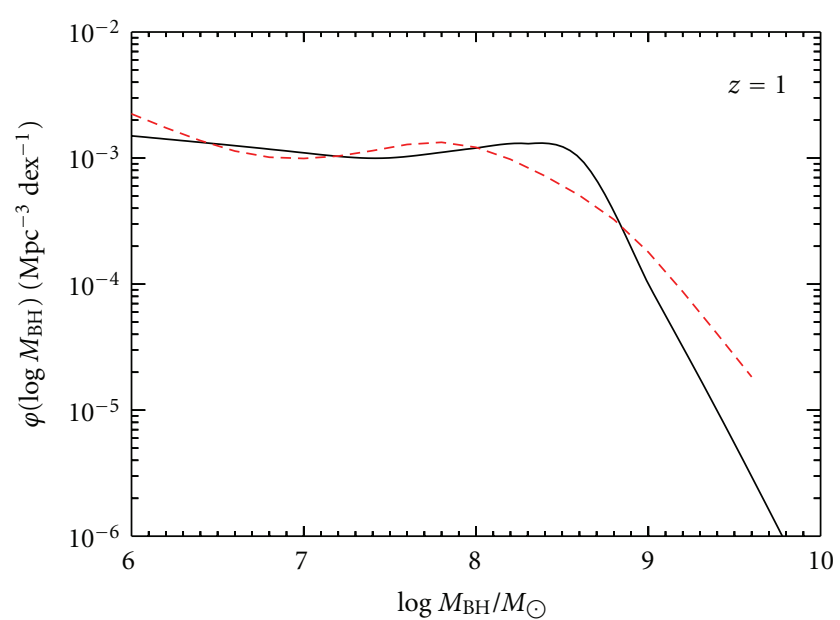

(a)

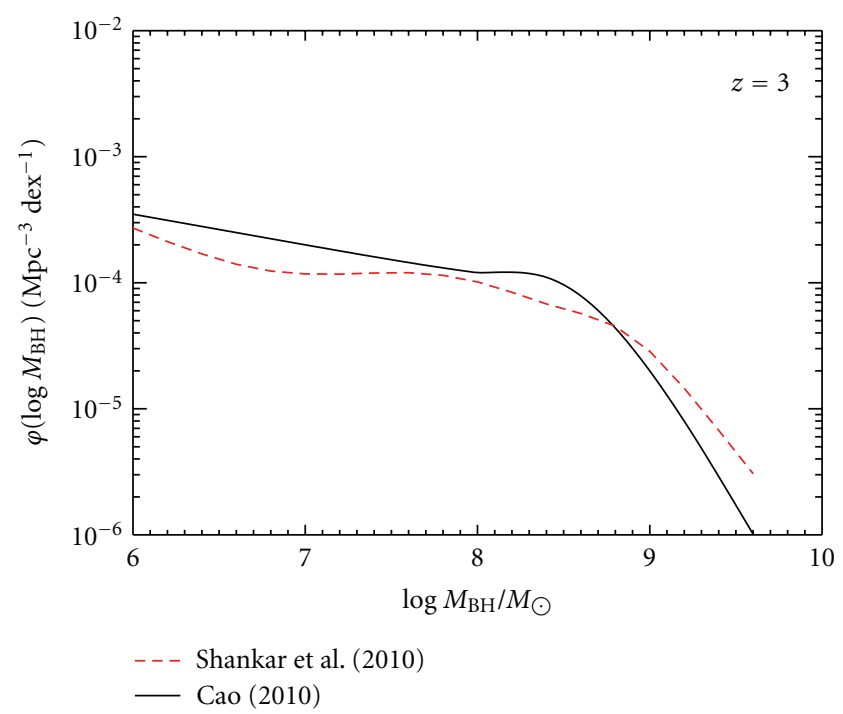

(c)

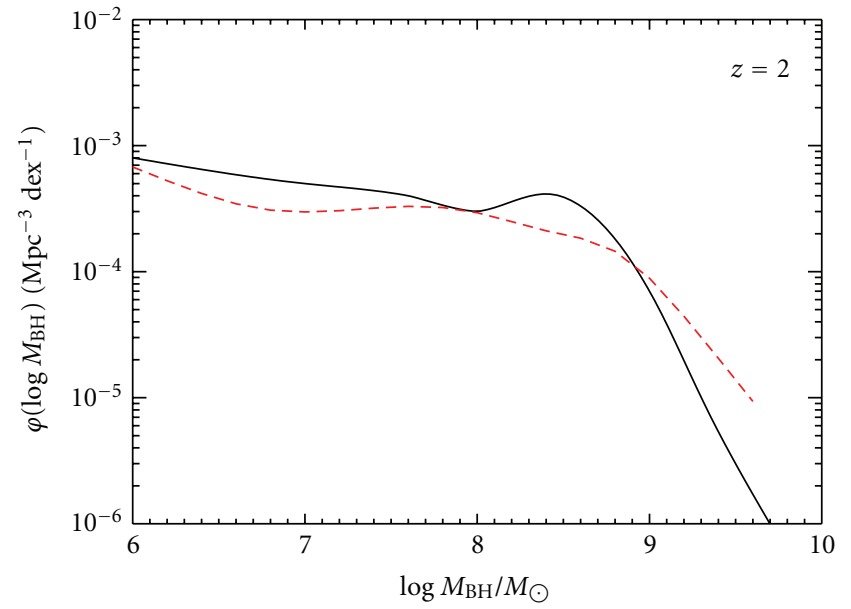

(b)

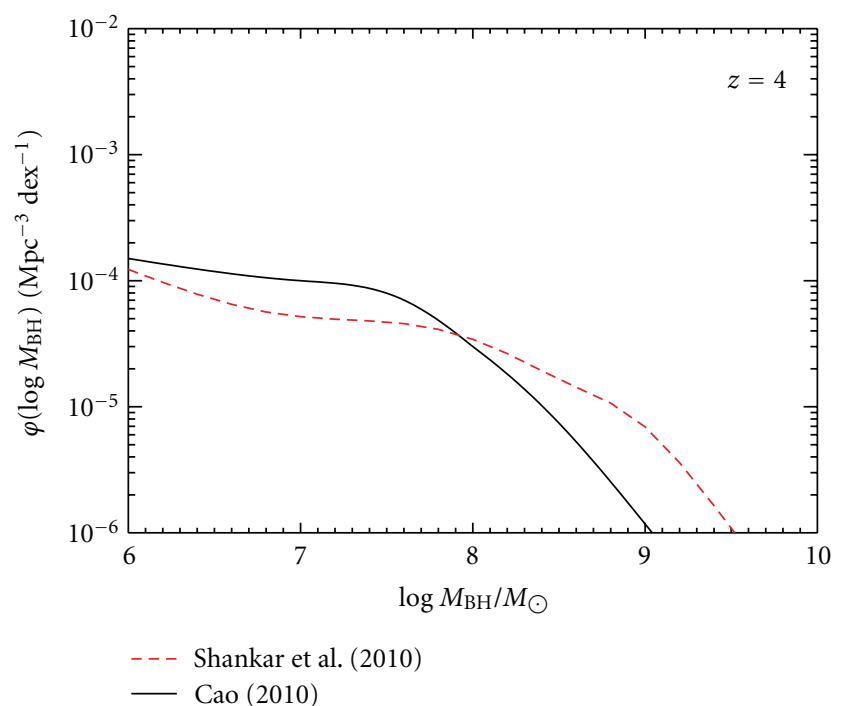

(d)

Figure 3: Comparison of two recently estimated BHMFs, calculated by Shankar et al. [64] (red dashed line) with that calculated by Cao [92] (solid black line). Both BHMFs were estimated by assuming a quasar lightcurve, where Shankar et al. [64] used a step function model while Cao [92] assumed a power-law decay. Despite the two different models, the BHMFs are similar at a variety of redshifts, except at possibly the high-mass end.

into a power-law decay. Cao [92] also modeled the quasar lightcurve as undergoing a power-law decay. Lightcurves undergoing a power-law decay arise from self-regulation models and describe the evolution of the lightcurve after black hole feedback unbinds the accreting gas, therefore quenching its fuel supply. The power-law decay occurs either as a result of evolution of a blast wave $[36,99]$ or from viscous evolution of the accretion disk [100,101].

In Figure 3, we compare the BHMF calculated by Shankar et al.[64] with that calculated by Cao [92]. For Shankar et al. [64], we show their reference model, which assumes a radiative efficiency of $\epsilon_{r}=0.065$, an accretion rate relative to Eddington of $\dot{M} / \dot{M}_{\text {Edd }}=0.6$, and that half of all SMBHs are active at $z=6$. We show the model from Cao [92] which assumes a radiative efficiency of $\epsilon_{r}=0.11$ and a quasar lifetime of $7.5 \times 10^{8} \mathrm{yr}$, as it better matches the Shankar et al. [64] estimates. The two estimates of the BHMF agree fairly well, despite the different quasar lightcurve models.

In general, most of the studies that have used (6) in combination with an assumed quasar lightcurve have concluded the following.

(i) Most SMBH growth occurs in periods when the quasar is radiating near the Eddington limit.

(ii) Most, if not all, of the local black hole mass function can be explained as the relic of previous AGN activity, implying that mergers of SMBHs are not important for building up the local mass function. 
(iii) SMBH growth is antihierarchical, with the most massive black holes growing first. This has also been termed "downsizing" of active SMBHs.

(iv) The lifetime of AGN activity is $\sim$ a few $\times 10^{8} \mathrm{yr}$.

(v) Most SMBHs have nonzero spin, as implied by inferred radiative efficiencies of $\epsilon_{r} \gtrsim 0.06$.

However, while (6) has proven to be an important tool for studying SMBH growth and estimating the black hole mass function, it must be kept in mind that the use of (6) often entails some strong assumptions. These methods rely on the assumed form of the quasar lightcurve, distribution of radiative efficiencies, and bolometric corrections, all of which are subject to considerable uncertainty. Moreover, in general, these methods also rely on an estimate of the local black hole mass function, which, as discussed in Section 3, is itself subject to considerable uncertainty. Indeed, there is a strong degeneracy between the estimated radiative efficiency of accretion and the normalization of the local BHMF, and therefore the uncertainty in $\epsilon_{r}$ is linearly proportional to that in the normalization (or integral) of the local BHMF. All of these issues have the potential to introduce systematic error into methods based on (6), and further work is needed in reducing these systematics.

4.2. Methods That Include the Distribution of Active Supermassive Black Holes. An alternative to the methods described in Section 4.1 is to estimate the average value of the accretion rate onto SMBHs directly from the observational data. This avoids the issue of assuming a form for the quasar lightcurve, as instead $\left\langle L\left(M_{\mathrm{BH}}, t\right)\right\rangle$ is derived directly from the estimated distribution of $L / L_{\mathrm{Edd}}$. Techniques based on this approach require a means of linking the mass function of active SMBHs to observational quantities, which is done via scaling relationships. This was the approach of Merloni [89] and Merloni and Heinz [63], who employed the black hole "fundamental plane" (BHFP) [40, 41].

The BHFP is a scaling relationship between $M_{\mathrm{BH}}$, radio luminosity, and X-ray luminosity, that exists for lowaccretion rate black holes (i.e., $\dot{M} / \dot{M}_{\text {Edd }} \lesssim 0.01$ ), extending from galactic black holes to supermassive ones. The BHFP likely reflects the connection between $M_{\mathrm{BH}}$ and the conversion of the accretion flow into radiative energy and jet power. It, in principle, enables one to connect the radio and X-ray luminosity functions to the mass function of active SMBHs. Having obtained a distribution of $M_{\mathrm{BH}}$ and X-ray luminosity at a given redshift for the active SMBH population, Merloni [89] and Merloni and Heinz [63] then convert this to a joint distribution of $M_{\mathrm{BH}}$ and $\dot{M}_{\mathrm{acc}}$ assuming a conversion from X-ray luminosity to $\dot{M}_{\text {acc }}$ which depends on the Eddington ratio. The joint distribution of $M_{\mathrm{BH}}$ and $\dot{M}_{\mathrm{acc}}$ at a given redshift for active SMBHs therefore enables calculation of the average growth rate $\left\langle\dot{M}\left(M_{\mathrm{BH}}, t(z)\right)\right\rangle$, which can then be combined with the continuity equation to calculate the black hole mass function at the next redshift. Their estimated BHMF is shown in Figure 4, which is a recreation of their Figure 5. Similar to methods based on assuming a quasar lightcurve, Merloni and Heinz [63] concluded that
SMBHs grow antihierarchically; however, in contrast to the lightcurve methods, Merloni and Heinz [63] concluded that most SMBHs have low spin as inferred from their derived radiative efficiency. In addition, Merloni and Heinz [63] concluded that the distribution of SMBH accretion rates is broad and that most $\mathrm{SMBH}$ growth occurs during a radiatively efficient accretion mode.

The method of estimating the BHMF from the BHFP developed by Merloni [89] and Merloni and Heinz [63] has the advantage that it derives the distribution of accretion rates empirically. However, there are also disadvantages to this approach. The uncertainties regarding the bolometeric correction, estimation of the local BHMF, and radiative efficiency also apply to the BHFP method as well. Moreover, as discussed in Merloni and Heinz [63], the BHFP is only defined for low-accretion rate objects, that is, objects with $L / L_{\mathrm{Edd}} \lesssim 10^{-2}$. Merloni and Heinz [63] extrapolate the BHFP to higher accretion rates, after rescaling the normalization to ensure that the radio luminosity is weak for AGN in the radiatively efficient mode (those objects with $L / L_{\mathrm{Edd}} \gtrsim 10^{-2}$ and lacking a jet). Unfortunately, the AGN in the radiatively efficient mode make a significant contribution to the X-ray luminosity function, from which $\left\langle\dot{M}\left(M_{\mathrm{BH}}, t\right)\right\rangle$ is derived. Moreover, most studies, including those based on the BHFP, have concluded that most SMBH growth occurs at $L / L_{\mathrm{Edd}} \gtrsim 10^{-2}$, which corresponds to the radiatively efficient mode. Because the radiatively efficient mode also corresponds to the regime of largest systematic uncertainty for the BHFP, there is the potential for significant systematic error in estimating the BHMF based on the BHFP, as well as in estimating the primary mode of $\mathrm{SMBH}$ growth. There is thus a need for further improvement to our understanding of the scaling relationships involving $M_{\mathrm{BH}}$ and the AGN SED.

\section{Black Hole Mass Functions of AGN}

Thus far, we have focused on methods for estimating the mass function of all SMBHs. In this section, we will describe methods for estimating the BHMF for those SMBHs in AGN and the results that have come from the application of these methods.

5.1. Methods Based on Scaling Relationships Involving the Broad Emission Lines. The steady improvement in reverberation mapping of AGN $[102,103]$ has revealed a correlation between the luminosity of AGN and the broad-line region radius $[104,105]$. It is therefore possible, in principle, to obtain an estimate of $M_{\mathrm{BH}}$ for broad-line AGN (BLAGN) by combining a luminosity-based estimate of the broad-line region size with an estimate of the velocity dispersion of the broad-line region gas obtained from the width of the broad emission lines [47]. These virial mass estimates are then calibrated to the estimates of $M_{\mathrm{BH}}$ obtained from reverberation mapping, which themselves are calibrated to be consistent with the local $M_{\mathrm{BH}^{-}} \sigma_{*}$ relationship [106, 107]. Currently, calibrations exist for $\mathrm{H} \alpha$ [108], $\mathrm{H} \beta$ (e.g., [48]), Mg-II [109111], and C IV [48]. The statistical scatter in the virial mass estimates is currently estimated to be $\sim 0.4 \mathrm{dex}$ [48], 


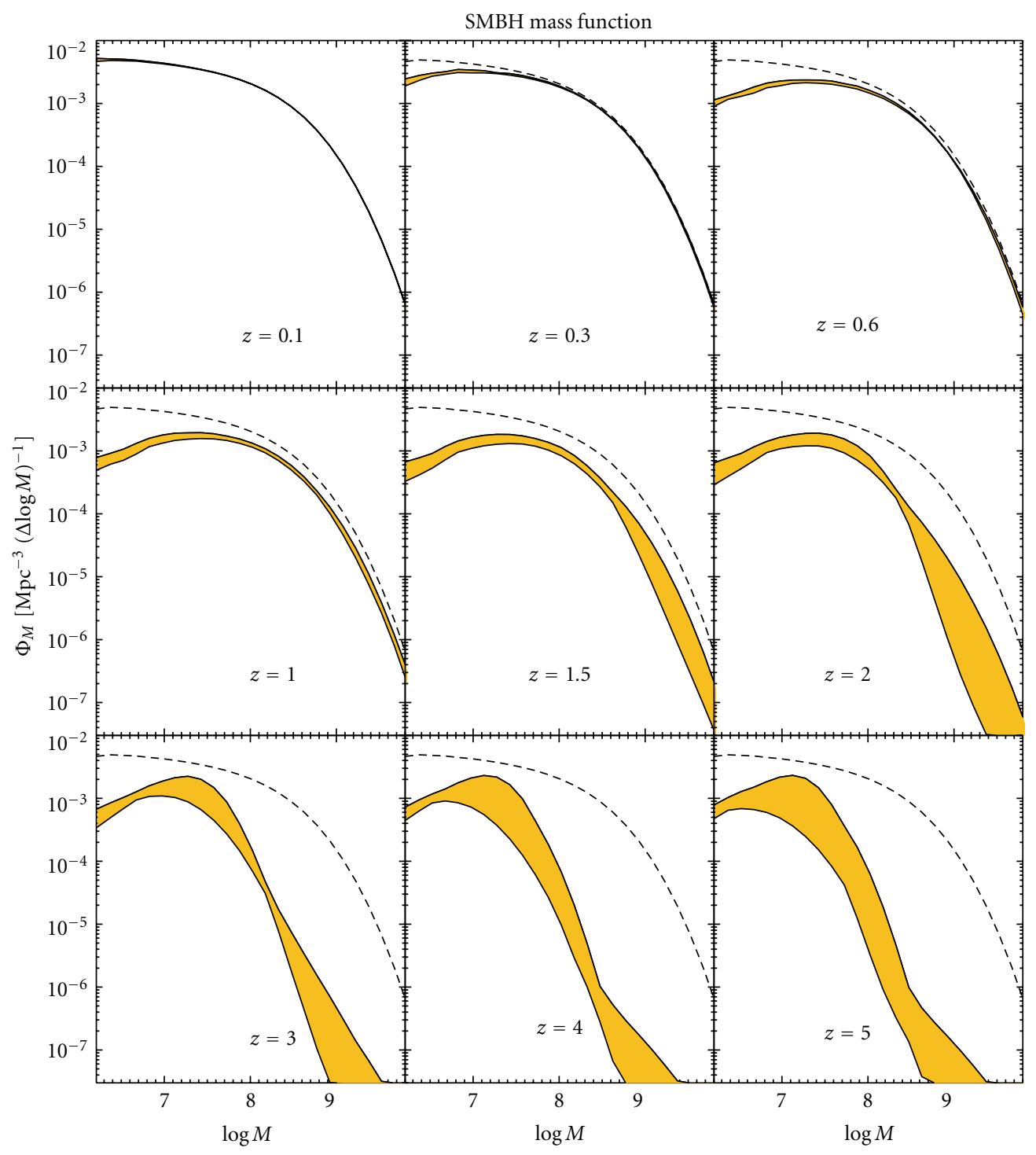

FIGURE 4: Figure 5 from Merloni and Heinz [63], showing the redshift evolution of their estimated BHMF. The dashed line is the local BHMF and the shaded regions reflect the uncertainty in the BHMF that is due to uncertainties in the AGN luminosity function. The high mass end of their estimated BHMF is built up faster than the low-mass end, a phenomenon that has been called "downsizing".

although there are indications that the scatter may be smaller, at least for the most luminous quasars [112-116]. Moreover, it should be noted that the calibration for $\mathrm{Mg}$ II is obtained by enforcing consistency in the mean values of the Mg II mass estimator and the $\mathrm{H} \beta$ and $\mathrm{C}$ IV ones, and therefore there is currently no direct estimate of the statistical scatter in $\mathrm{Mg}$ II-based virial mass estimates. In contrast, the amplitudes of the statistical scatter for $\mathrm{H} \beta$ and $\mathrm{C}$ IV are estimated by comparing mass estimates derived from these lines with the masses derived from reverberation mapping [48]. Although there is currently very little reverberation mapping data for C IV, the estimate of the dispersion in the C IV-based mass estimates should not be biased so long as the masses based on reverberation mapping are reliable estimates of the true $M_{\mathrm{BH}}$, regardless of which emission line was used in the reverberation mapping campaign.
Early estimates of the mass function of SMBHs in BLAGN were obtained by binning up the virial mass estimates and applying a $1 / V_{\max }$ correction [110, 117-119], a technique borrowed from luminosity function estimation. Greene and Ho [118] estimated the local BHMF for BLAGN from the SDSS DR4, while Vestergaard et al. [119] estimated the BHMF for BLAGN over $0.3<z<5$ using the uniformly selected quasar sample from the SDSS DR3 [120]. Vestergaard and Osmer [110] estimated the BHMF for the brightest BLAGN using objects from a variety of surveys, as their sample was designed to complement the uniformly selected SDSS DR3 sample. Unfortunately, as discussed in Section 2.1, this method of binning up the mass estimates suffers from biases due to the large statistical scatter in the virial mass estimates, and due to the inability of a luminositybased $1 / V_{\max }$ correction to correct for incompleteness in 


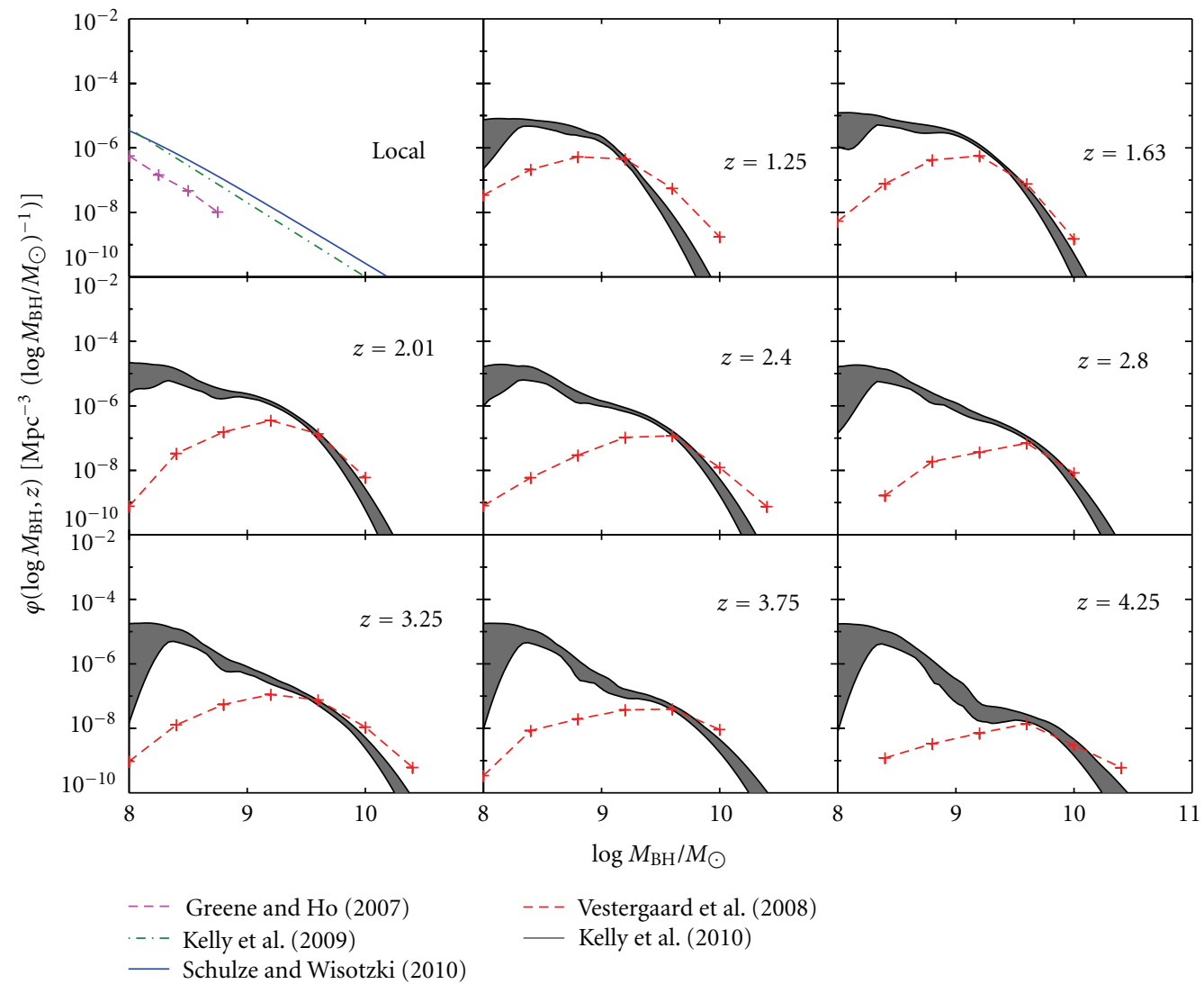

FIGURE 5: Broad-line AGN BHMFs at a variety of redshifts. Shown are the local BHMF estimated by Greene and Ho [118] (dashed magenta line), Kelly et al. [46] (dot-dashed green line), and Schulze and Wisotzki [121] (solid blue line). Also shown are the $z>1$ BHMFs estimated by Vestergaard et al. [119] (dashed red line) and Kelly et al. [115] (shaded region), where the shaded region for the Kelly et al. [115] estimate defines an approximate 95\% confidence region. The BHMFs estimated by Greene and Ho [118] and Vestergaard et al. [119] are flux-limited BHMFs, as they did not fully correct for incompleteness in $M_{\mathrm{BH}}$.

$M_{\mathrm{BH}}$. Subsequent attempts have further improved in their methodology, providing more accurate BHMFs.

Shen et al. [113] employed a forward-modeling approach where the mass function and Eddington ratio distribution were estimated by matching the observed distribution of mass estimates and luminosity to that implied by the model BHMF and Eddington ratio distribution. Their method accounts for incompleteness and the statistical scatter in the mass estimates but lacked statistical rigor in that the matching was done visually. Schulze and Wisotzki [121] employed a maximum-likelihood technique for estimating the local BHMF for BLAGN. Their method corrects for incompleteness in $M_{\mathrm{BH}}$ but does not correct the BHMF for the broadening caused by the statistical scatter in the virial mass estimates. Kelly et al. [46] developed a Bayesian method that corrects for both the statistical scatter in the mass estimates and incompleteness and used their method to estimate the local BHMF of BLAGN from the Bright Quasar Survey [122]. Kelly et al. [115] used the method of [46] to estimate the BHMF of BLAGN at $1<z<4.5$ from the mass estimates in the SDSS DR3 quasar sample [119]. The BLAGN BHMFs from a variety of studies are compiled in Figure 5, showing the evolution of the BHMF from the local universe out to $z=4.5$. More recently, Shen and Kelly [116] extended the Bayesian method of [46] to include a possible luminosity-dependent bias in virial mass estimates derived from the emission line FWHM, the existence of which was suggested by Shen and Kelly [77]. Shen and Kelly [116] applied their method to the SDSS DR7 uniformly-selected quasar sample, independently estimating the BHMF and Eddington ratio distribution in different redshifts bins.

Similar to the methods based on the continuity equation, investigations of the BHMF for BLAGN have found evidence for the anti-hierarchical growth of SMBHs, that is, cosmic "down-sizing" of BLAGN activity. The inferred Eddington ratio distributions are wide, and the density of SMBHs continues to increase toward Eddington ratios which are below the survey completeness limit. In addition, Kelly et al. [115] used the BLAGN BHMF to estimate the lifetime of broadline quasar activity to be $t_{\mathrm{BL}} \sim 150 \mathrm{Myr}$ among SMBHs with $M_{\mathrm{BH}} \sim 10^{9} M_{\odot}$, which is similar to quasar lifetimes inferred from the continuity equation. Kelly et al. [115] also used their estimated BHMF to estimate the maximum mass of a SMBH to be $M_{\mathrm{BH}} \sim 3 \times 10^{10} M_{\odot}$, which is in agreement with theoretical expectations $[123,124]$. 
Mass functions estimated from scaling relationships for BLAGN have the advantage that they are derived from estimates of $M_{\mathrm{BH}}$ that are obtained for individual sources, providing a more "direct" estimate of the mass function than those based on the continuity equation. However, they have the disadvantage that they are only available for a subset of the AGN population, which itself is only a subset of the $\mathrm{SMBH}$ population. This complicates comparison with other $\mathrm{SMBH}$ mass functions, as the fraction of AGN with broad emission lines is poorly constrained, especially as a function of mass. This being said, BHMFs of BLAGN represent a subset of SMBHs that are actively growing at the time that they are observed, and, as the aforementioned studies have demonstrated, their mass function still contains important information on SMBH growth.

As with all methods of BHMF estimation, the virial mass estimates and the mass functions derived from them still suffer from systematics. First, there is the usual problem of calculating a bolometeric correction, although this only affects the estimated Eddington ratio distribution and not the BHMF. Second, there are a few concerns with the virial mass estimates which could introduce systematic error; some of these have been discussed by Greene and Ho [125]. For one, most of the reverberation mapping data is only available for the $\mathrm{H} \beta$ line. Because of the limited $\mathrm{Mg}$ II data, the Mg II scaling relationship is in general not calibrated using objects with black hole mass estimates from reverberation mapping. There may be systematic effects with luminosity or Eddington ratio when using the FWHM-based scaling relationships $[116,126]$, possibly due to a dependence of the broad-line region structure on these quantities. Systematic effects on broad-line region geometry, which can effect the inferred velocity dispersion, are a particular concern for C IV, which is thought to arise in an accretion disk wind [127]. Along these lines, unaccounting for radiation pressure on broad-line clouds may also bias the virial masses, especially among those AGN radiating near the Eddington limit [128]; however, its importance is still debated [129-131]. In addition, the reliability of line width measurements can rapidly deteriorate for low $S / N$ data [132]. And, finally, the BLAGN virial mass estimates are calibrated to the reverberation mapping derived masses, which themselves are calibrated to lie on the local $M_{\mathrm{BH}}-\sigma_{*}$ relationship. Most of the AGN that are used to calibrate the reverberation mapping masses to the $M_{\mathrm{BH}}-\sigma_{*}$ relationship have lower masses and are hosted by late-type galaxies, for which there is evidence that the $M_{\mathrm{BH}}-\sigma_{*}$ relationship begins to break down [81]. Greene et al. [81] argue that the normalization of the scaling relationships inferred when limiting the calibration to low-mass SMBHs hosted in late-type galaxies may be about a factor of $\sim 1.5$ lower than that used for the current broad-line mass estimates [81]. However, dynamical mass estimates exist for two reverberation mapped AGN: NGC 3227 [133, 134] and NGC 4151 $[134,135]$. In both cases, the masses derived from dynamical modeling and reverberation mapping agree, so it is unclear if a smaller scaling factor is needed for late-type galaxies. These issues show that there are still many remaining questions regarding virial masses, highlighting the need for further study using high-quality reverberation mapping data.
5.2. Other Methods for Estimating the Black Hole Mass Function of $A G N$. Before broad-line mass estimates, there were two earlier attempts at estimating the BHMF for AGN, which we briefly mention here. Siemiginowska and Elvis [136] and Hatziminaoglou et al. [137] used a model for the AGN lightcurve arising due to thermal-viscous accretion disk instabilities [138] to calculate the expected distribution of luminosity at a given black hole mass. Based on this calculated distribution, they used the quasar luminosity function to constrain the quasar black hole mass function. Siemiginowska and Elvis [136] found evidence for SMBH downsizing in AGN, consistent with later work.

Franceschini et al. [139] found a tight correlation between $M_{\mathrm{BH}}$ and the total radio power observed in a sample of local galaxies. They then used their empirical relationship to estimate the local BHMF derived from the local radio luminosity function of galaxies. While many of the objects in their sample are not considered AGN in the traditional sense, Fraceschini et al. [139] argue that this correlation is a signature of an advection-dominated accretion flow, thought to dominate at low accretion rates relative to Eddington. Therefore, while these SMBHs may not be "active" in the quasar sense, the determination of their mass function relies on radio emission from the $\mathrm{SMBH}$ accretion flow, so this method may still be considered a method for estimating the BHMF for active SMBHs. Franceschini et al. [139] compared their BHMF to models of AGN activity and found that it was inconsistent with AGN activity being continuous and long lived, but consistent with AGN activity being transient and possibly recurrent.

\section{Theoretical Models for Black Hole Mass Functions across Cosmic Time}

There have been numerous theoretical models for the formation and growth of supermassive black holes, and coevolution with their host galaxies. Understanding this formation, growth, and coevolution is one of the current most important outstanding issues in extragalactic astrophysics. Because the black hole mass function provides a census of the $\mathrm{SMBH}$ population and its evolution, it is one of the most fundamental observational quantities available for constraining models of SMBH formation and growth. As such, many theoretical investigations have predicted a BMHF for comparison with the empirical BHMF. In this section, we review some of the models for SMBH formation and growth. There have been numerous theoretical models for SMBH growth and formation, and it is beyond the scope of this primarily empirically-focused review to review all of them; instead, we focus on those theoretical models that predict a BHMF.

6.1. Modeling the Coevolution of SMBHs and Galaxies: Predicted BHMFs. Early models for the coevolution of SMHBs and galaxies linked the growth of black holes to the properties of host dark matter halos, with periods of SMBH growth occurring in quasar phases initiated by mergers. In general, early studies that predicted a BHMF used various prescriptions to relate $M_{\mathrm{BH}}$ to the mass of the host halo [26, 140143]. More recent models for the coevolution of SMBHs 
and galaxies has incorporated AGN feedback from the SMBH. In addition, the availability of empirical BHMFs have enabled modelers to compare their more recent models with observational data. In general, the models are qualitatively in agreement with the empirical results, in that they are able to match the local BHMF fairly well and predict downsizing of SMBHs. However, considering the current systematic and statistical uncertainties in the empirical results, it is difficult to place rigorous empirical constraints on the models such that certain models may be ruled out. Because of this, we simply summarize some of the different recent models that have been developed which predict the BHMF.

Granato et al. [144] developed a model incorporating feedback from AGN and supernovae, where the feeding of the SMBH is driven by stellar radiation drag on gas. Their predicted local BHMF agrees with that estimated by Shankar et al. [57]. Cattaneo et al. [30] used halo merger trees constructed from $\mathrm{N}$-body simulations to track the growth of SMBHs. In their model, the black hole fueling rate was proportional to the star formation rate of the host galaxy burst component and the density of the cold gas in the starburst component. Their model predicted SMBH downsizing, with the most massive part of the BHMF being built up first, in agreement with the subsequent empirical studies.

Hopkins et al. [145] describe a model for the coevolution of SMBHs and galaxies whereby all major mergers of gasrich galaxies trigger a quasar. In this model, the final black hole mass is assumed to be on average proportional to the host spheroidal mass, in agreement with the local scaling relationships between SMBHs and their host galaxies. Hopkins et al. [145] estimated the merger rate of gas-rich galaxies by combining theoretical constraints of the halo and subhalo mass functions with empirical constraints on halo occupation models. Their model also predicts SMBH downsizing, and their predicted BHMF matches the local BHMF derived by Marconi et al. [53]. Similarly, Shen [146] also assumed that quasars are triggered by major mergers of gas-rich galaxies, with the SMBHs growing via accretion in these quasar phases. Shen [146] used a halo merger rate based on theoretical expectations from $N$-body simulations and assumed a universal quasar lightcurve shape having an exponential increase followed by a power law decay (see also [62]). The BHMF predicted by Shen [146] broadly agrees with the local one estimated by Shankar et al. [64] and predicts that most SMBHs with $M_{\mathrm{BH}}>3 \times 10^{8} M_{\odot}$ were in place by $z=1$ but only $50 \%$ of them were assembled by $z=2$.

Most recently, Fanidakis et al. $[147,148]$ extended the model of [23], which includes AGN feedback, to also follow the spin distribution of SMBHs. In their model, SMBHs are fueled through accretion of cold gas from mergers, disk instabilities, and cooling flows from hot halos. However, the inclusion of SMBH spin enabled them to include different radiative efficiencies, which dictates how much accreted material actually grows the black hole, and to provide an improved model for the amount of mechanical feedback imparted through an AGN jet, both of which depend on the spin of the black hole. Their model predicts that the presentday universe is dominated by SMBHs with $M_{\mathrm{BH}} \sim 10^{7}-$ $10^{8} M_{\odot}$, and that the BHMF at $M_{\mathrm{BH}}>10^{9} M_{\odot}$ was largely built up at $z<2$ due to an increase in both lower-accretion rate "radio-mode" growth and mergers of SMBHs.

Almost all models for the cosmological coevolution of SMBHs and galaxies that predict a BHMF have been of an analytical or semi-analytical nature. An exception is the study done by Di Matteo et al. [31], who present the results from cosmological hydrodynamic simulations of the $\Lambda$ CDM model that follow the growth of galaxies and SMBHs, including their feedback processes, at $z>1$. Direct cosmological simulations such as these should, in principle, provide the most accurate results as to the predicted BHMF, and for identifying the relevant physical processes that are important in shaping the BHMF. However, current cosmological hydrodynamic simulations suffer from the fact that they cannot resolve processes on physical scales corresponding to the SMBH accretion flow. In fact, Di Matteo et al. [31] use a gravitational softening length of $\epsilon=2.73 \mathrm{~h}^{-1} \mathrm{kpc}$. Instead, Di Matteo et al. [31] employ a subresolution model where the accretion onto the SMBH is estimated using a Bondi-HoyleLyttleton parameterization [149-151] with a correction factor to account for the fact that the Bondi radius is not resolved. They assume a radiative feedback energy efficiency of 5\% [20], which is the only free parameter in their model and required in order to match the normalization of the observed local $M_{\mathrm{BH}}-\sigma_{*}$ relationship. Their calculated BHMF at $z=1$ matches the local BHMF for $M_{\mathrm{BH}}>2 \times 10^{8} M_{\odot}$. In addition, Di Matteo et al. [31] also find downsizing in their model, in agreement with observations, with the high-mass end of the BHMF being largely in place by $z \sim 2$.

In Figure 6, we compile predicted BHMFs from several recent models for SMBH formation and growth [31, 145$147,152]$. In general, the models tend to agree to within a factor of a few with regards to the BHMF. However, they diverge at $M_{\mathrm{BH}} \gtrsim 10^{9} M_{\odot}$, where their predicted $\mathrm{SMBH}$ number densities can differ by over an order of magnitude.

6.2. Modeling the BHMF of SMBH Seeds. Recent work has made improvement to models for the BHMF by focusing on theoretical modeling of the distribution of seed SMBHs. The discovery of quasars at $z \approx 6-7$ with $M_{\mathrm{BH}} \sim 10^{9} M_{\odot}$ [153, 154] places strong constraints on the formation of SMBH seeds due to the very limited amount of time available at that redshift to grow SMBHs (e.g., [155]). Lodato and Natarajan [156] derive the BHMF of SMBH seeds at $z \sim 15$ that are the result of the collapse of pregalactic disks which have not yet been enriched by metals [157]. Black holes formed through such a mechanism have masses $M_{\mathrm{BH}} \sim 10^{5} M_{\odot}$, while black holes which are the remnants of Pop III stars have $M_{\mathrm{BH}} \sim$ $10^{3} M_{\odot}$. A similar seed black hole formation mechanism is through "quasistars" [158-160], which are also able to produce seed black holes with $M_{\mathrm{BH}} \sim 10^{5} M_{\odot}$.

Volonteri et al. [161] describe a model for the growth of SMBHs seeded according to the direct collapse model of Lodato and Natarajan [157] with varying formation efficiencies. In addition, they also compared the results from this model using SMBHs seeded from Pop III remnants. Volonteri et al. [161] grow SMBHs through major mergers, and force the black hole mass after the galaxy merger to scale with the circular velocity of the host halo; additional growth is also 


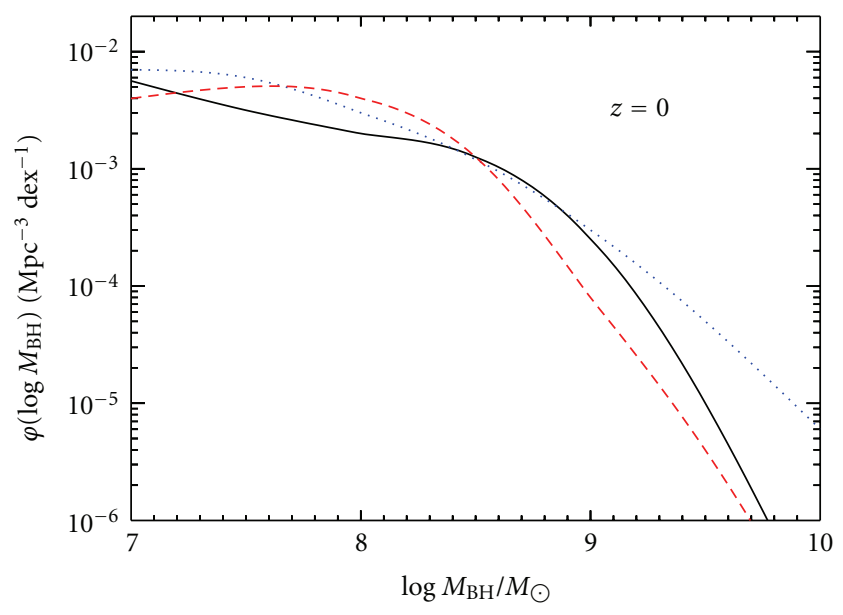

(a)

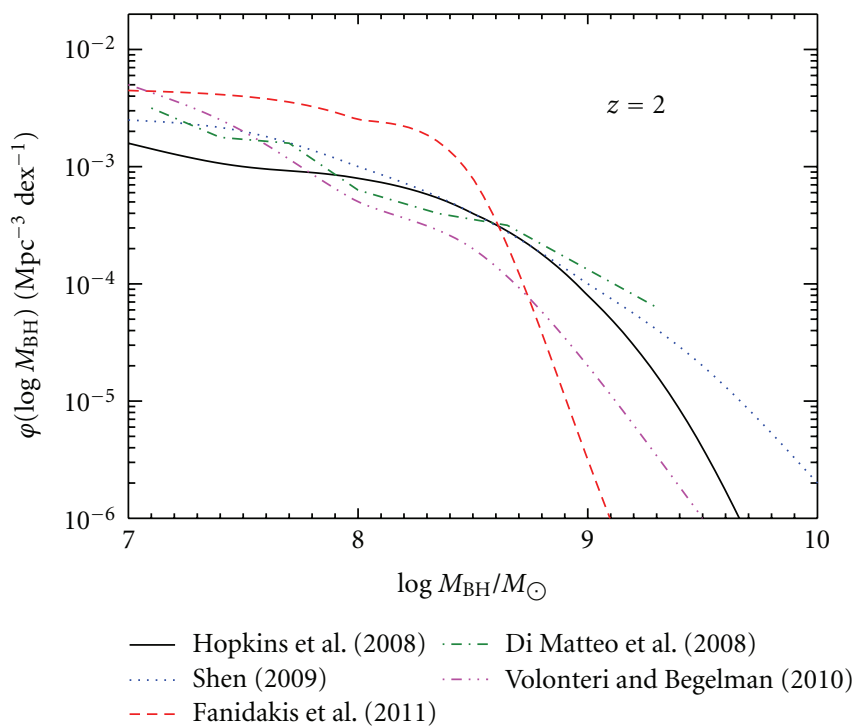

(c)

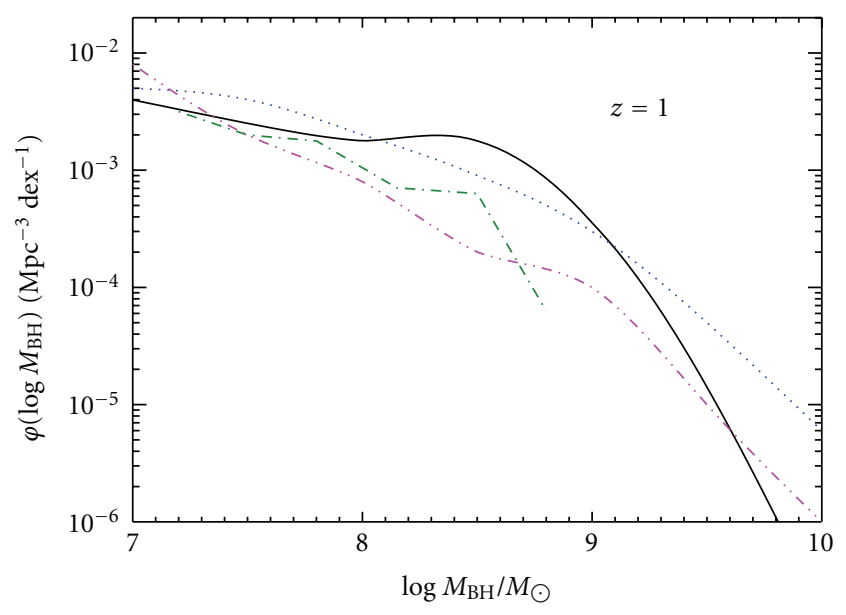

(b)

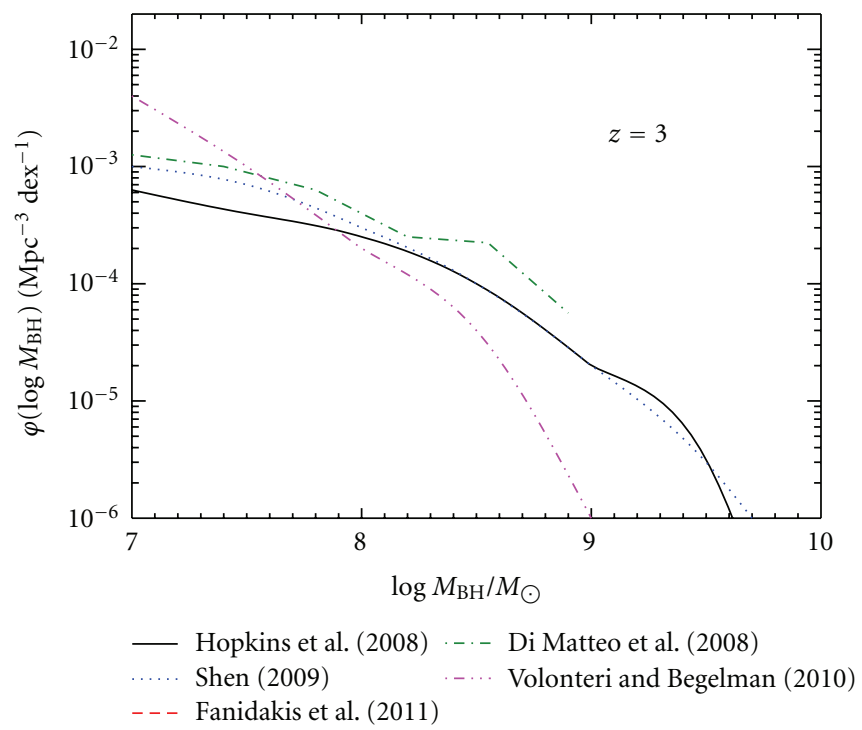

(d)

FIguRE 6: Compilation of BHMFs predicted by several recent models for SMBH formation and growth. Shown are the BHMFs predicted by Hopkins et al. [145] (solid black line), Shen [146] (dotted blue line), Fanidakis et al. [147] (dashed red line), Di Matteo et al. [31] (dot-dashed green line), and Volonteri and Begelman [152] (dashed-dotted-dotted-dotted magenta line). In general the number densities predicted by the models agree to within a factor of a few, although they diverge at $M_{\mathrm{BH}} \gtrsim 10^{9} M_{\odot}$. Some of these authors did not report BHMFs at each redshift shown, so we only show those available at each redshift.

provided through black hole mergers. Their merger trees are based on a Monte Carlo algorithm based on the extended Press-Schechter formalism. They find that most significant differences in the local BHMF with respect to black hole formation efficiency occur at $M_{\mathrm{BH}}<10^{7} M_{\odot}$, with the number density of SMBHs with $M_{\mathrm{BH}}<10^{7} M_{\odot}$ increasing with increasing formation efficiency. Volonteri and Begelman [152] performed a similar analysis as that of Volonteri et al. [161] but instead used SMBH seeds formed via quasistars. The BHMFs calculated by Volonteri and Begelman [152] match those of Merloni and Heinz [63] at the high-mass end, at least at $z>2$.

Natarajan and Volonteri [162] used a growth and seeding model which is very similar to that employed by Volonteri et al. [161]. However, they also predict the BHMF for broad line quasars, assuming that $20 \%$ of quasars are unobscured. They compare the BHMF derived from their model at $1<$ $z<4.5$ to the BHMF for broad line quasars reported by Kelly et al. [115], and to the BHMF for all SMBHs reported by Merloni and Heinz [63]. Natarajan and Volonteri [162] concluded that seeds from Pop III stars have difficulty reproducing the BLAGN BHMF, especially at high redshift, while seeds resulting from the direct collapse of pregalactic disks do better at fitting the high mass end of the BLAGN BHMF at $z>2$.

Lippai et al. [163] studied the impact that the fraction of halos that form a SMBH seed at $z>6$ has on the local BHMF. They are able to reproduce the observed quasar luminosity 
function and local BHMF for a suitable range of radiative efficiency and quasar lifetime, so long as at least $\approx 10 \%$ of high- $z$ halos contained SMBH seeds. Tanaka and Haiman [164] present BHMFs at $z=6$ based on a comprehensive exploration of the parameter space governing the buildup of the $z>6$ BHMF, including models for SMBH seeds that form from Pop III remnants and through direct collapse, variations in the occupation fraction, and a detailed treatment of gravitational recoil. Comparing their predicted BHMFs to observations of $z \sim 6$ quasars from the SDSS, they concluded that $\sim 100 M_{\odot}$ seeds can grow into the SMBHs observed at $z \sim 6$, so long as they are nearly continuously embedded in dense gas, form at $z>30$, have low occupation fractions, and stop forming by $z \sim 20$.

\section{Directions for Future Work and Improvement}

Before concluding this paper, we present a discussion of possible future empirical and theoretical work relevant to BHMF studies. These include the following.

\subsection{Better Characterization of the SMBH-Host Galaxy Scaling} Relationships. Currently, the local BHMF is estimated from the distribution of host galaxy properties assuming that $M_{\mathrm{BH}}$ has a constant log-normal scatter about a singlepower law scaling relationship. As discussed in Section 3, recent observations have provided reason to doubt this assumption, suggesting that the correlations break down at the highest and lowest masses. This will create biases in the BHMF determined from the scaling relationships, which in turn will also affect the BHMF estimated from the continuity equation. Further direct $M_{\mathrm{BH}}$ estimates from dynamical and kinematic modeling should be obtained for a variety of galaxy types, especially at the high- and low$M_{\mathrm{BH}}$ end. The next class of $25+\mathrm{m}$ telescopes should provide a significantly improved picture of the scaling relationships, thus providing us with more accurate estimates of the local BHMF.

\subsection{Improvements to Techniques Based on the Continuity} Equation. Most studies that have invoked the continuity equation to link the local BHMF to the AGN luminosity function have assumed a single radiative efficiency, which is equivalent to assuming a single black hole spin, and a universal AGN lightcurve. Neither of these assumptions are likely to be true, and improvements to this type of modeling should include a distribution of SMBH spin and AGN lightcurves. In addition, we need to better characterize the bolometeric corrections, which remain a significant source of systematic uncertainty. The continuity equation techniques should also be extended to map the evolution of the full joint 3-dimensional distribution of black hole mass, accretion rate, and spin. While this will not necessarily have a direct effect on estimating the BHMF, it will provide insight into the dominant accretion modes experienced by active $\mathrm{SMBH}$ and into the dominant fueling mechanism for AGN activity, as the spin distribution traces the $\mathrm{SMBH}$ fueling history [165].
7.3. Better Characterization of Scaling Relationships Involving $M_{\mathrm{BH}}$ for $A G N$. The dominant scaling relationship for estimating $M_{\mathrm{BH}}$ in AGN involves the broad emission lines. However, as discussed in Section 5.1, a number of systematic uncertainties remain. In order to reduce these systematics, we need to better understand the broad-line region geometry for the different emission lines and how it scales with luminosity, which will provide us with a more accurate conversion from line width to velocity dispersion. Moreover, accurate characterization of the broad-line region geometry should remove the need to calibrate the scaling relationships to the local $M_{\mathrm{BH}^{-}} \sigma_{*}$ relationship, which has its own set of systematics. Improvements to reverberation mapping campaigns and modeling [166-170], as well as increasing the number of AGN monitored for reverberation mapping, will be needed in order to really understand the systematics involved in the broad line mass estimates.

There is also the need to better characterize the black hole fundamental plane. Because the BHFP describes how the emission mechanisms responsible for the radio and X-ray flux scale with $M_{\mathrm{BH}}$, the BHFP coefficients depend on these emission mechanisms. However, these emission mechanisms depend on the geometry of the accretion state and the existence of a jet, which in turn depends on the accretion rate [171], and therefore the BHFP coefficients will be different for different classes of AGN [172-174]. In particular, the BHFP is currently poorly constrained for "soft-state" galactic black holes and radio-quiet AGN. Therefore, to reduce the systematics involved with the BHFP, it will be necessary to characterize the scaling relationships and their scatter for radio-quiet objects. A correlation between the radio and Xray luminosity has been observed for radio-quiet objects [175], implying that a BHFP should also exist for these objects. In order to better characterize the BHFP for radioquiet objects it will be necessary to obtain radio detections for a well-defined sample of radio-quiet AGN with reliable $M_{\mathrm{BH}}$ estimates and X-ray detections.

Finally, there has recently been the discovery of scaling relationships involving $M_{\mathrm{BH}}$ and the optical [176, 177] and $\mathrm{X}$-ray $[42,45,49]$ variability properties of AGN. Mass estimators based on these scaling relationships have not been rigorously developed yet, nor have they seen widespread use. However, the existence of these scaling relationships implies that the variability properties may offer another avenue for estimating $M_{\mathrm{BH}}$ and BHMFs, which may become increasingly valuable in the era of current and future large timedomain surveys, such as Pan-Starrs and LSST.

7.4. Understanding the Redshift Evolution of Scaling Relations. From the theoretical point of view, it is clear that highredshift scaling relations (or the lack thereof) between SMBH and their hosts provide unique and powerful constraints to models for AGN feeding and feedback, which cannot be otherwise distinguished (see, e.g., Merloni et al. [74] and references therein).

In practical terms, a better understanding of the evolution of scaling relations may also be very advantageous for BHMF studies. As we discussed above, current technique for BH mass estimation at $z>0$ involve unobscured, broad 
emission line QSOs. One can argue that, as long as we are restricted to just this class of QSOs, we will have to make critical assumptions about the properties of a significant part of the population to draw conclusions about the full BHMF (if we wanted, e.g., to compare with "continuity-equationbased" methods). On the other hand, large multiwavelength surveys do and will provide a wealth of information on the host galaxies of obscured AGN at high redshift, that represent the numerically dominant part of the growing black holes population [178-181]. Therefore, if we had an independent way to put constraints on the nature of the $\mathrm{BH}$-host relation for these objects, we could explore the uncharted territory of BHMF for obscured AGN (and for the entire population). Such an independent information could come, for example, from IR studies of broad emission lines which could act as probes of the $\mathrm{BH}$ potential less affected by obscuration. The first exploratory works pursuing this line of research have recently been published, for example, [182, 183].

From the technical point of view, a lot of work of course is needed to better understand how reliable these estimators are. Another big "technical" challenge of all studies of the evolution of scaling relations is the fact that they require a thorough assessment of the many observational biases one encounters in studying high redshift AGN and their hosts [184].

7.5. Accounting for Black Hole Kicks in Theoretical Models. Many theoretical models for the BHMF do not include recoiling effects caused by the merger of two black holes. However, recent theoretical work on black hole recoils suggests that black holes can spend a significantly large enough amount of time offset from the central region of the host galaxy to alter their growth, thereby increasing the scatter about the scaling relationships and decreasing the final black hole mass [185-188]. On the other hand, Volonteri et al. [189] and Volonteri et al. [190] find that ejected SMBHs are rare at $z<5$, especially for massive SMBHs, suggesting that accounting for ejected black holes will not make a significant difference in the BHMF. Moreover, Tanaka and Haiman [164] find that recoiling black holes only modestly effect the BHMF. Further improvement in our understanding of the effects and frequency of black hole recoil will ensure the accurate implementation of black hole recoil into models for $\mathrm{SMBH}$ growth.

7.6. Improvements to Our Understanding of AGN Feedback. Most current theoretical models for SMBH growth involve AGN feedback and assume a single efficiency for coupling feedback energy to the gas; this feedback efficiency is usually treated as a free parameter. An improved physical understanding of AGN feedback will improve theoretical models for the BHMF, as the feedback efficiency affects the dynamics of the SMBH's fuel supply and therefore the amount that the $\mathrm{SMBH}$ accretes as a function of redshift. Recent high-resolution hydrodynamic simulations in one dimension [191193] and two dimensions [194, 195] have concluded that AGN feedback efficiency increases with the Eddington ratio and that the values are below the value of $\sim 5 \%$ assumed in many current theoretical models for SMBH growth. Further improvements to simulations developed for studying AGN feedback will lead to a better physical understanding of AGN feedback, which will improve theoretical models for SMBH growth and the BHMF.

On the observational side, future X-ray observations should provide considerable improvement in our understanding of AGN feedback. X-ray spectra are needed in order to determine the total column density of the gas, and thus its kinetic energy flux, which can be compared to the energy output of the SMBH. Current X-ray observations from Chandra have found evidence that AGN feedback exists in the local universe [196]. However, X-ray calorimeters on future X-ray satellites will be needed for further improvement as they provide the high throughput and spectral resolution needed to measure column densities and velocities of ionized gas, and consequently the kinetic energy flux, in a large sample of AGN across a broad redshift range.

7.7. Improvements in Resolution and Subresolution Modeling for Direct Hydrodynamic Simulations. Full hydrodynamic cosmological simulations offer the most promising avenue for providing a physically motivated BHMF without free parameters and for unambiguously identifying the relevant physical processes in building up the BHMF. However, they currently cannot resolve scales relevant to the accretion flow onto the SMBH. Numerical codes based on adaptive mesh refinement techniques will provide improvement in resolution, but it will likely be a while before hydrodynamic cosmological simulations are able to follow SMBH growth in large cosmological volumes while simultaneously resolving the scales relevant for individual black holes. In the meantime, further improvement can be made to the subresolution modeling employed by current hydrodynamic simulations.

One way of improving current sub-resolution models may be to implement the results on AGN feedback based on the type of work described in the previous bullet point. Another improvement is in modifying the sub-resolution model for the SMBH accretion flow. Current methods assume the Bondi rate combined with a correction factor to account for the fact that the temperature and density of the gas are not resolved at the Bondi radius. Not surprisingly, the growth of the $\mathrm{SMBH}$ is sensitive to how this correction factor is modeled [33]. Moreover, sub-resolution models based on the Bondi rate neglect the angular momentum of the gas, and thus the Bondi rate may not be representative of the actual accretion rate onto the SMBH. Hopkins and Quataert [197] used high-resolution hydrodynamic simulations to conclude that the Bondi rate was a poor estimate of the actual accretion rate onto the $\mathrm{SMBH}$, and describe a sub-resolution model which accurately estimated the actual accretion rate in their simulations. In addition, Power et al. [198] suggest an alternative sub-resolution model based on an "accretion particle" to provide a more accurate estimate of the black hole accretion rate. The implementation of improved subresolution models for accretion rate and feedback into cosmological hydrodynamic simulations, as well as further improvements to the sub-resolution models, will result in more accurate predicted BHMFs, allowing a more insightful comparison with empirical BHMFs. 


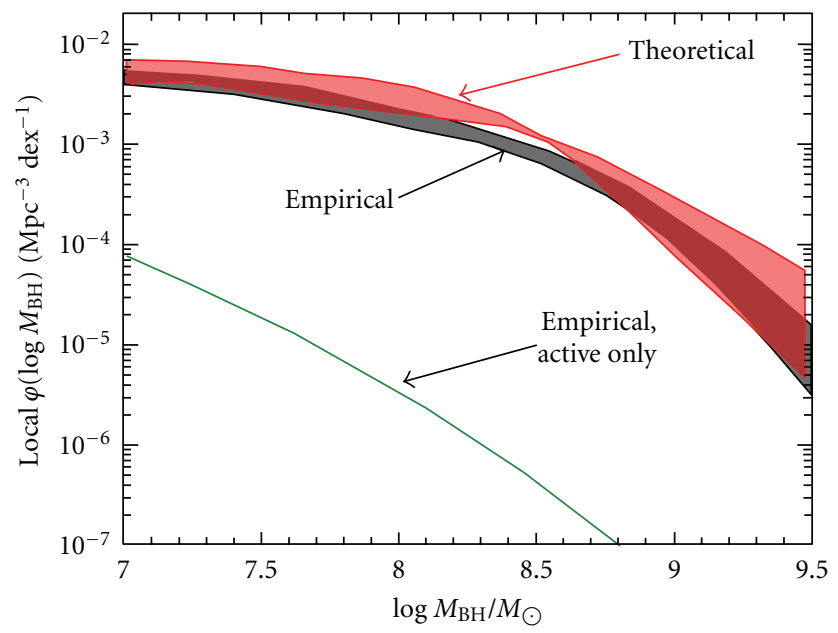

(a)

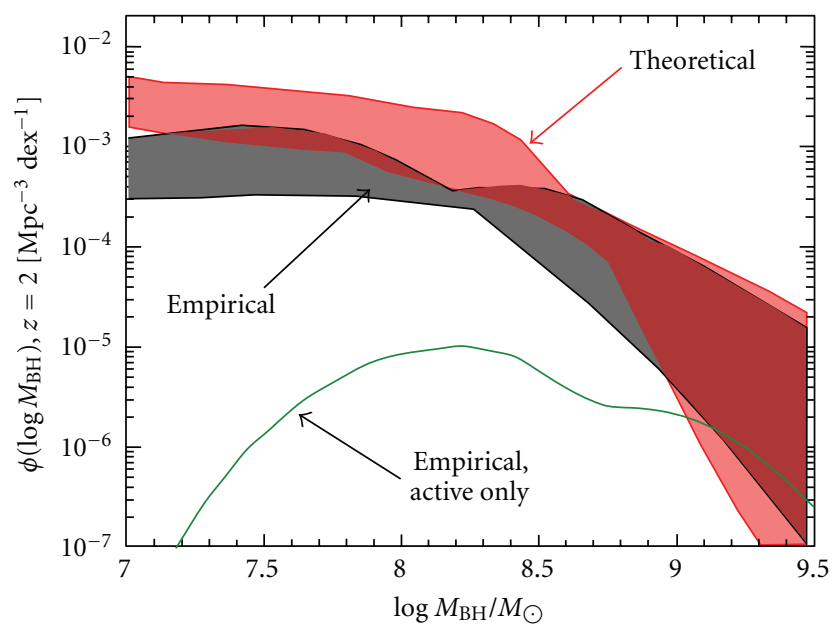

(b)

FIgURE 7: Comparison of empirical estimates of the BHMF (grey shaded region) with BHMFs predicted by theoretical models for SMBH formation and growth (red shaded region), in both the local universe (a) and at $z=2$ (b). Also shown are the estimated BHMFs of broad-line AGN only (solid green line), from Schulze and Wisotzki [121] (a) and Kelly et al. [115] (b). The empirical estimates of the BHMF are those shown in Figures 3 and 4, while the theoretical estimates are those shown in Figure 6. The shaded regions define the spread in the estimates and models and may be considered to be a crude estimate of their uncertainty. In general, the theoretical number densities are consistent with the empirical ones to within a factor of a few.

In this paper we have reviewed current estimates of the SMBH mass function, as well as theoretical models for the BHMF. As discussed above, each of the methods for estimating the BHMF has their own set of systematics. In Figure 7 , we compare the empirical estimates of the local BHMF (defined by the shaded region in Figure 2) with the BHMFs predicted by the theoretical models compiled in Figure 6 . In addition, in Figure 7, we also compare the empirical BHMFs at $z=2$, as estimated using the lightcurve method (shown in Figure 3) and the black hole fundamental plane (shown in Figure 4), with BHMFs predicted by the theoretical models. In both Figures we also include the BHMFs for broad-line AGN as estimated by Schulze and Wisotzki [121] and Kelly et al. [115]. In general, the theoretical models are consistent to within a factor of a few with the empirical estimates of the BHMF, although there is a large spread in the models and empirical estimates at $z=2$. Moreover, the estimated number densities of broad-line AGN are significantly lower than those of all SMBHs, suggesting that only a small fraction of SMBHs are active across a broad range in $M_{\mathrm{BH}}$, except for possibly SMBHs at $z \sim 2$ with $M_{\mathrm{BH}} \gtrsim 10^{9} M_{\odot}$.

Despite the differences in the methods for estimating the BHMF and the theoretical models, they have lead to a number of common conclusions. In particular, the empirical results have presented a picture whereby $\mathrm{SMBHs}$ grow primarily via accretion in active phases (Eddington ratios $L_{\text {bol }} / L_{\mathrm{Edd}}>0.01$ ), that quasar activity is a relatively shortlived phenomenon relative to the lifetime of the SMBH and host galaxy (i.e., small "duty cycles" for AGN activity) and that $\mathrm{SMBH}$ growth is anti-hierarchical with the most massive end of the BHMF being built up first. These empirical results are qualitatively in agreement with the steadily improving theoretical models.

\section{Acknowledgments}

The authors would like to thank Tommaso Treu, Priya Natarajan, Marta Volonteri, Aneta Siemiginowska, Xiaohui Fan, Marianne Vestergaard, Alister Graham, and Zoltán Haiman for helpful comments. In addition, they would like to thank two anonymous referees whose comments improved our paper. B. C. Kelly acknowledges support by NASA through Hubble Fellowship Grant no. HF-51243.01 awarded by the Space Telescope Science Institute, which is operated by the Association of Universities for Research in Astronomy, Inc., for NASA, under Contract NAS 5-26555.

\section{References}

[1] J. Kormendy and D. Richstone, "Inward bound-the search for supermassive black holes in galactic nuclei," Annual Review of Astronomy and Astrophysics, vol. 33, no. 1, pp. 581-624, 1995.

[2] R. J. McLure and J. S. Dunlop, "The black hole masses of Seyfert galaxies and quasars," Monthly Notices of the Royal Astronomical Society, vol. 327, no. 1, pp. 199-207, 2001.

[3] R. J. McLure and J. S. Dunlop, "On the black hole-bulge mass relation in active and inactive galaxies," Monthly Notices of the Royal Astronomical Society, vol. 331, no. 3, pp. 795-804, 2002.

[4] K. Gebhardt, R. Bender, G. Bower et al., "A relationship between nuclear black hole mass and galaxy velocity dispersion," Astrophysical Journal, vol. 539, no. 1, pp. L13-L16, 2000.

[5] D. Merritt and L. Ferrarese, "The M. $-\sigma$ relation for supermassive black holes," Astrophysical Journal, vol. 547, no. 1, pp. 140-145, 2001.

[6] S. Tremaine, K. Gebhardt, R. Bender et al., "The slope of the black hole mass versus velocity dispersion correlation," Astrophysical Journal, vol. 574, no. 2, pp. 740-753, 2002. 
[7] A. W. Graham, P. Erwin, N. Caon, and I. Trujillo, "A correlation between galaxy light concentration and supermassive black hole mass," Astrophysical Journal, vol. 563, no. 1, pp. L11-L14, 2001.

[8] A. W. Graham and S. P. Driver, "A log-quadratic relation for predicting supermassive black hole masses from the host bulge sérsic index," Astrophysical Journal Letters, vol. 655, no. 1, pp. 77-87, 2007.

[9] J. Magorrian, S. Tremaine, D. Richstone et al., "The demography of massive dark objects in galaxy centers," Astronomical Journal, vol. 115, no. 6, pp. 2285-2305, 1998.

[10] A. Marconi and L. K. Hunt, "The relation between black hole mass, bulge mass, and near-Infrared luminosity," Astrophysical Journal Letters, vol. 589, no. 1, pp. L21-L24, 2003.

[11] N. Häring and H.-W. Rix, "On the black hole mass-bulge mass relation," Astrophysical Journal Letters, vol. 604, no. 2, pp. L89-L92, 2004.

[12] M. C. Aller and D. O. Richstone, "Host galaxy bulge predictors of supermassive black hole mass," Astrophysical Journal, vol. 665, no. 1, pp. 120-156, 2007.

[13] P. F. Hopkins, L. Hernquist, T. J. Cox, B. Robertson, and E. Krause, "A theoretical interpretation of the black hole fundamental plane," Astrophysical Journal, vol. 669, no. 1, pp. 45-66, 2007.

[14] J. Silk and M. J. Rees, "Quasars and galaxy formation," Astronomy and Astrophysics, vol. 331, no. 2, pp. L1-L4, 1998.

[15] A. C. Fabian, "The obscured growth of massive black holes," Monthly Notices of the Royal Astronomical Society, vol. 308, no. 4, pp. L39-L43, 1999.

[16] M. C. Begelman and B. B. Nath, "Self-regulated black hole accretion, the M- $\sigma$ relation and the growth of bulges in galaxies," Monthly Notices of the Royal Astronomical Society, vol. 361, no. 4, pp. 1387-1392, 2005.

[17] N. Murray, E. Quataert, and T. A. Thompson, "On the maximum luminosity of galaxies and their central black holes: feedback from momentum-driven winds," Astrophysical Journal, vol. 618, no. 2, pp. 569-585, 2005.

[18] C. Y. Peng, "How mergers may affect the mass scaling relation between geuvitationally bound systems," Astrophysical Journal Letters, vol. 671, no. 2, pp. 1098-1107, 2007.

[19] K. Jahnke and A. V. Macciò, "The non-causal origin of the black-hole-galaxy scaling relations," Astrophysical Journal Letters, vol. 734, no. 2, article 92, 2011.

[20] T. Di Matteo, V. Springel, and L. Ilernquist, "Energy input from quasars regulates the growth and activity of black holes and their host galaxies," Nature, vol. 433, no. 7026, pp. 604 $607,2005$.

[21] V. Springel, T. Di Matteo, and L. Hernquist, "Black holes in galaxy mergers: the formation of red elliptical galaxies," Astrophysical Journal, vol. 620, no. 2, pp. L79-L82, 2005.

[22] P. H. Johansson, T. Naab, and A. Burkert, "Equal- and unequal-mass mergers of disk and elliptical galaxies with black holes," Astrophysical Journal, vol. 690, no. 1, pp. 802821, 2009.

[23] R. G. Bower, A. J. Benson, R. Malbon et al., "Breaking the hierarchy of galaxy formation," Monthly Notices of the Royal Astronomical Society, vol. 370, no. 2, pp. 645-655, 2006.

[24] D. J. Croton, V. Springel, S. D. M. White et al., "The many lives of active galactic nuclei: cooling flows, black holes and the luminosities and colours of galaxies," Monthly Notices of the Royal Astronomical Society, vol. 365, no. 1, pp. 11-28, 2006.

[25] M. G. Haehnelt, P. Natarajan, and M. J. Rees, "High-redshift galaxies, their active nuclei and central black holes," Monthly
Notices of the Royal Astronomical Society, vol. 300, no. 3, pp. 817-827, 1998.

[26] G. Kauffmann and M. Haehnelt, "A unified model for the evolution of galaxies and quasars," Monthly Notices of the Royal Astronomical Society, vol. 311, no. 3, pp. 576-588, 2000.

[27] M. G. Haehnelt and G. Kauffmann, "The correlation between black hole mass and bulge velocity dispersion in hierarchical galaxy formation models," Monthly Notices of the Royal Astronomical Society, vol. 318, no. 3, pp. L35-L38, 2000.

[28] J. S. B. Wyithe and A. Loeb, "Self-regulated growth of supermassive black holes in galaxies as the origin of the optical and X-ray luminosity functions of quasars," Astrophysical Journal, vol. 595, no. 2, pp. 614-623, 2003.

[29] M. Volonteri, F. Haardt, and P. Madau, "The assembly and merging history of supermassive black holes in hierarchical models of galaxy formation," Astrophysical Journal, vol. 582, no. 2, pp. 559-573, 2003.

[30] A. Cattaneo, J. Blaizot, J. Devriendt, and B. Guiderdoni, "Active galactic nuclei in cosmological simulations -I. Formation of black holes and spheroids through mergers," Monthly Notices of the Royal Astronomical Society, vol. 364, no. 2, pp. 407-423, 2005.

[31] T. Di Matteo, J. Colberg, V. Springel, L. Hernquist, and D. Sijacki, "Direct cosmological simulations of the growth of black holes and galaxies," Astrophysical Journal, vol. 676, no. 1, pp. 33-53, 2008.

[32] R. S. Somerville, P. F. Hopkins, T. J. Cox, B. E. Robertson, and L. Hernquist, "A semi-analytic model for the co-evolution of galaxies, black holes and active galactic nuclei," Monthly Notices of the Royal Astronomical Society, vol. 391, no. 2, pp. 481-506, 2008.

[33] C. M. Booth and J. Schaye, "Cosmological simulations of the growth of supermassive black holes and feedback from active galactic nuclei: method and tests," Monthly Notices of the Royal Astronomical Society, vol. 398, no. 1, pp. 53-74, 2009.

[34] F. Marulli, E. Branchini, L. Moscardini, and M. Volonteri, "Modelling active galactic nuclei: ongoing problems for the faint-end of the luminosity function," Monthly Notices of the Royal Astronomical Society, vol. 375, no. 2, pp. 649-656, 2007.

[35] L. Ciotti and J. P. Ostriker, "Cooling flows and quasars. II. Detailed models of feedback-modulated accretion flows," Astrophysical Journal, vol. 551, no. 1, pp. 131-152, 2001.

[36] P. F. Hopkins and L. Hernquist, "Fueling low-level AGN activity through stochastic accretion of cold gas," Astrophysical Journal Supplement Series, vol. 166, no. 1, pp. 1-36, 2006.

[37] O. Guyon, D. B. Sanders, and A. Stockton, "Near-infrared adaptive optics imaging of QSO host galaxies," Astrophysical Journal Supplement Series, vol. 166, no. 1, pp. 89-127, 2006.

[38] J. M. Gabor et al., "Active galactic nucleus host galaxy morphologies in COSMOS," The Astrophysical Journal, vol. 691, no. 1, pp. 705-722, 2009.

[39] A. Georgakakis, A. L. Coil, E. S. Laird et al., "Host galaxy morphologies of X-ray selected AGN: assessing the significance of different black hole fuelling mechanisms to the accretion density of the Universe at $\mathrm{z} \sim 1$," Monthly Notices of the Royal Astronomical Society, vol. 397, no. 2, pp. 623-633, 2009.

[40] A. Merloni, S. Heinz, and T. Di Matteo, "A fundamental plane of black hole activity," Monthly Notices of the Royal Astronomical Society, vol. 345, no. 4, pp. 1057-1076, 2003.

[41] H. Falcke, E. Körding, and S. Markoff, "A scheme to unify low-power accreting black holes. Jet-dominated accretion 
flows and the radio/X-ray correlation," Astronomy and Astrophysics, vol. 414, no. 3, pp. 895-903, 2004.

[42] I. M. McHardy, E. Koerding, C. Knigge, P. Uttley, and R. P. Fender, "Active galactic nuclei as scaled-up Galactic black holes," Nature, vol. 444, no. 7120, pp. 730-732, 2006.

[43] S. W. Davis and A. Laor, "The radiative efficiency of accretion flows in individual active galactic nuclei," Astrophysical Journal Letters, vol. 728, no. 2, article 98, 2011.

[44] M. A. Sobolewska, A. Siemiginowska, and M. Gierliński, "Simulated spectral states of active galactic nuclei and observational predictions," Monthly Notices of the Royal Astronomical Society, vol. 413, no. 3, pp. 2259-2268, 2011.

[45] B. C. Kelly, M. Sobolewska, and A. Siemiginowska, "A stochastic model for the luminosity fluctuations of accreting black holes," Astrophysical Journal Letters, vol. 730, no. 1, article 52, 2011.

[46] B. C. Kelly, M. Vestergaard, and X. Fan, "Determining quasar black hole mass functions from their broad emission lines: application to the bright quasar survey," The Astrophysical Journal, vol. 692, article 1388, 2009.

[47] A. Wandel, B. M. Peterson, and M. A. Malkan, "Central masses and broad-line region sizes of active galactic nuclei. I. Comparing the photoionization and reverberation techniques," Astrophysical Journal, vol. 526, no. 2, pp. 579-591, 1999.

[48] M. Vestergaard and B. M. Peterson, "Determining central black hole masses in distant active galaxies and quasars. II. Improved optical and UV scaling relationships," Astrophysical Journal, vol. 641, no. 2, pp. 689-709, 2006.

[49] M. Nikolajuk, I. E. Papadakis, and B. Czerny, "Black hole mass estimation from X-ray variability measurements in active galactic nuclei," Monthly Notices of the Royal Astronomical Society, vol. 350, no. 2, pp. L26-L30, 2004.

[50] X.-L. Zhou, S.-N. Zhang, D.-X. Wang, and L. Zhu, "Calibrating the correlation between black hole mass and X-ray variability amplitude: X-ray only black hole mass estimates for active galactic nuclei and ultra-luminous X-ray sources," Astrophysical Journal Letters, vol. 710, no. 1, pp. 16-23, 2010.

[51] K. Gültekin, D. O. Richstone, K. Gebhardt et al., "The M- $\sigma$ and $\mathrm{M}-\mathrm{L}$ relations in galactic bulges, and determinations of their intrinsic scatter," Astrophysical Journal, vol. 698, no. 1, pp. 198-221, 2009.

[52] B. C. Kelly, X. Fan, and M. Vestergaard, "A flexible method of estimating luminosity functions," Astrophysical Journal, vol. 682, no. 2, pp. 874-895, 2008.

[53] A. Marconi, G. Risaliti, R. Gilli, L. K. Hunt, R. Maiolino, and M. Salvati, "Local supermassive black holes, relics of active galactic nuclei and the X-ray background," Monthly Notices of the Royal Astronomical Society, vol. 351, no. 1, pp. 169-185, 2004.

[54] P. Salucci, E. Szuszkiewicz, P. Monaco, and L. Danese, "Mass function of dormant black holes and the evolution of active galactic nuclei," Monthly Notices of the Royal Astronomical Society, vol. 307, no. 3, pp. 637-644, 1999.

[55] Q. Yu and S. Tremaine, "Observational constraints on growth of massive black holes," Monthly Notices of the Royal Astronomical Society, vol. 335, no. 4, pp. 965-976, 2002.

[56] M. C. Aller and D. Richstone, "The cosmic density of massive black holes from galaxy velocity dispersions," Astronomical Journal, vol. 124, no. 6, pp. 3035-3041, 2002.

[57] F. Shankar, P. Salucci, G. L. Granato, G. De Zotti, and L. Danese, "Supermassive black hole demography: the match between the local and accreted mass functions," Monthly
Notices of the Royal Astronomical Society, vol. 354, no. 4, pp. 1020-1030, 2004.

[58] T. R. Lauer, S. M. Faber, D. Richstone et al., "The masses of nuclear black holes in luminous elliptical galaxies and implications for the space density of the most massive black holes," Astrophysical Journal, vol. 662, no. 2, pp. 808-834, 2007.

[59] A. W. Graham, S. P. Driver, P. D. Allen, and J. Liske, "The Millennium Galaxy Catalogue: the local supermassive black hole mass function in early- and late-type galaxies," Monthly Notices of the Royal Astronomical Society, vol. 378, no. 1, pp. 198-210, 2007.

[60] A. W. Graham and S. P. Driver, "The local supermassive black hole mass density: corrections for dependencies on the Hubble constant," Monthly Notices of the Royal Astronomical Society, vol. 380, no. 1, pp. L15-L19, 2007.

[61] E. Tundo, M. Bernardi, J. B. Hyde, R. K. Sheth, and A. Pizzella, "On the inconsistency between the black hole mass function inferred from M.- $\sigma$ and M.-L correlations," Astrophysical Journal, vol. 663, no. 1, pp. 53-60, 2007.

[62] Q. Yu and Y. Lu, "Toward precise constraints on the growth of massive black holes," Astrophysical Journal Letters, vol. 689, no. 2, pp. 732-754, 2008.

[63] A. Merloni and S. Heinz, "A synthesis model for AGN evolution: supermassive black holes growth and feedback modes," Monthly Notices of the Royal Astronomical Society, vol. 388, no. 3, pp. 1011-1030, 2008.

[64] F. Shankar, D. H. Weinberg, and J. Miralda-Escudé, "Selfconsistent models of the AGN and black hole populations: duty cycles, accretion rates, and the mean radiative efficiency," Astrophysical Journal Letters, vol. 690, no. 1, pp. 2041, 2009.

[65] M. Vika, S. P. Driver, A. W. Graham, and J. Liske, "The Millennium Galaxy Catalogue: the Mbh-Lspheroid derived supermassive black hole mass function," Monthly Notices of the Royal Astronomical Society, vol. 400, no. 3, pp. 1451-1460, 2009.

[66] N. Tamura, K. Ohta, and Y. Ueda, "Supermassive black hole mass functions at intermediate redshifts from spheroid and AGN luminosity functions," Monthly Notices of the Royal Astronomical Society, vol. 365, no. 1, pp. 134-146, 2006.

[67] F. Shankar, M. Bernardi, and Z. Haiman, "The evolution of the $\mathrm{MBH}-\sigma$ relation inferred from the age distribution of local early-type galaxies and active galactic nuclei evolution," Astrophysical Journal Letters, vol. 694, no. 2, pp. 867-878, 2009.

[68] Z. Haiman, L. Ciotti, and J. P. Ostriker, "Reasoning from fossils: learning from the local black hole population about the evolution of quasars," Astrophysical Journal Letters, vol. 606, no. 2, pp. 763-773, 2004.

[69] Z. Haiman, R. Jimenez, and M. Bernardi, "Reconstructing the cosmic evolution of quasars from the age distribution of local early-type galaxies," Astrophysical Journal Letters, vol. 658, no. 2, pp. 721-730, 2007.

[70] T. Treu, M. A. Malkan, and R. D. Blandford, "The relation between black hole mass and velocity dispersion at $\mathrm{z} \sim 0.37$," Astrophysical Journal, vol. 615, no. 2, pp. L97-L100, 2004.

[71] C. Y. Peng, C. D. Impey, H. W. Rix et al., "Probing the coevolution of supermassive black holes and galaxies using gravitationally lensed quasar hosts," Astrophysical Journal, vol. 649, no. 2, pp. 616-634, 2006.

[72] T. Treu, J. H. Woo, M. A. Malkan, and R. D. Blandford, "Cosmic evolution of black holes and spheroids. II. Scaling 
relations at $\mathrm{z}=0.36$, , Astrophysical Journal, vol. 667, no. 1, pp. 117-130, 2007.

[73] J. H. Woo, T. Treu, M. A. Malkan, and R. D. Blandford, "Cosmic evolution of black holes and spheroids. III. The M $\mathrm{BH}-\sigma *$ relation in the last six billion years," Astrophysical Journal, vol. 681, no. 2, pp. 925-930, 2008.

[74] A. Merloni, A. Bongiorno, M. Bolzonella et al., "On the cosmic evolution of the scaling relations between black holes and their host galaxies: broad-line active galactic nuclei in the zCosmos survey," Astrophysical Journal, vol. 708, no. 1, pp. 137-157, 2010.

[75] V. N. Bennert, T. Treu, J. H. Woo et al., "Cosmic evolution of black holes and spheroids. IV. the M BH-L sph relation," Astrophysical Journal, vol. 708, no. 2, pp. 1507-1527, 2010.

[76] T. R. Lauer, S. Tremaine, D. Richstone, and S. M. Faber, "Selection bias in observing the cosmological evolution of the M- $\sigma$ - and M-L relationships," Astrophysical Journal, vol. 670, no. 1, pp. 249-260, 2007.

[77] Y. Shen and B. C. Kelly, "The impact of the uncertainty in single-epoch virial black hole mass estimates on the observed evolution of the black hole-bulge scaling relations," Astrophysical Journal Letters, vol. 713, no. 1, pp. 41-45, 2010.

[78] R. K. Sheth, M. Bernardi, P. L. Schechter et al., "The velocity dispersion function of early-type galaxies," Astrophysical Journal, vol. 594, no. 1, pp. 225-231, 2003.

[79] M. Bernardi, R. K. Sheth, E. Tundo, and J. B. Hyde, "Selection bias in the M. $-\sigma$ and M.-L correlations and its consequences," Astrophysical Journal, vol. 660, no. 1, pp. 267275, 2007.

[80] A. W. Graham, "Fundamental planes and the barless MBH- $\sigma$ relation for supermassive black holes," Astrophysical Journal Letters, vol. 680, no. 1, pp. 143-153, 2008.

[81] J. E. Greene, C. Y. Peng, M. Kim et al., "Precise black hole masses from megamaser disks: black hole-bulge relations at low mass," Astrophysical Journal Letters, vol. 721, no. 1, pp. 26-45, 2010.

[82] J. Hu, "The black hole mass-stellar velocity dispersion correlation: bulges versus pseudo-bulges," Monthly Notices of the Royal Astronomical Society, vol. 386, no. 4, pp. 2242-2252, 2008.

[83] J. E. Greene, L. C. Ho, and A. J. Barth, "Black holes in pseudobulges and spheroidals: a change in the black holebulge scaling relations at low mass," Astrophysical Journal, vol. 688, no. 1, pp. 159-179, 2008.

[84] A. W. Graham and I. H. Li, "The M bh- $\sigma$ diagram and the offset nature of barred active galaxies," Astrophysical Journal, vol. 698, no. 1, pp. 812-818, 2009.

[85] J. Kormendy, R. Bender, and M. E. Cornell, "Supermassive black holes do not correlate with galaxy disks or pseudobulges," Nature, vol. 469, no. 7330, pp. 374-376, 2011.

[86] A. W. Graham, C. A. Onken, E. Athanassoula, and F. Combes, "An expanded Mbh- $\sigma$ diagram, and a new calibration of active galactic nuclei masses," Monthly Notices of the Royal Astronomical Society, vol. 412, no. 4, pp. 2211-2228, 2011.

[87] A. Soltan, "Masses of quasars," Monthly Notices of the Royal Astronomical Society, vol. 200, pp. 115-122, 1982.

[88] Q. Yu and Y. Lu, "Constraints on QSO models from a relation between the QSO luminosity function and the local black hole mass function," Astrophysical Journal Letters, vol. 602, no. 2 I, pp. 603-624, 2004.

[89] A. Merloni, "The anti-hierarchical growth of supermassive black holes," Monthly Notices of the Royal Astronomical Society, vol. 353, no. 4, pp. 1035-1047, 2004.
[90] P. F. Hopkins, G. T. Richards, and L. Hernquist', “An observational determination of the bolometric quasar luminosity function," Astrophysical Journal, vol. 654, no. 2, pp. 731-753, 2007.

[91] X. Cao and F. Li, "Rapidly spinning massive black holes in active galactic nuclei: evidence from the black hole mass function," Monthly Notices of the Royal Astronomical Society, vol. 390, no. 2, pp. 561-566, 2008.

[92] X. Cao, "Cosmological evolution of massive black holes: Effects of eddington ratio distribution and quasar lifetime," Astrophysical Journal, vol. 725, no. 1, pp. 388-393, 2010.

[93] A. Cavaliere, P. Morrison, and K. Wood, "On Quasar evolution," Astrophysical Journal, vol. 170, pp. 223-231, 1971.

[94] T. A. Small and R. D. Blandford, "Quasar evolution and the growth of black holes," Monthly Notices of the Royal Astronomical Society, vol. 259, pp. 725-737, 1992.

[95] B. C. Kelly, J. Bechtold, J. R. Trump, M. Vestergaard, and M. Siemiginowska, "Observational constraints on the dependence of radio-quiet quasar X-ray emission on black hole mass and accretion rate," Astrophysical Journal Supplement Series, vol. 176, no. 2, pp. 355-373, 2008.

[96] R. V. Vasudevan and A. C. Fabian, "Piecing together the Xray background: bolometric corrections for active galactic nuclei," Monthly Notices of the Royal Astronomical Society, vol. 381, no. 3, pp. 1235-1251, 2007.

[97] R. V. Vasudevan, R. F. Mushotzky, L. M. Winter, and A. C. Fabian, "Optical-to-X-ray emission in low-absorption AGN: results from the Swift-BAT 9-month catalogue," Monthly Notices of the Royal Astronomical Society, vol. 399, no. 3, pp. 1553-1575, 2009.

[98] S. I. Raimundo and A. C. Fabian, "Eddington ratio and accretion efficiency in active galactic nuclei evolution," Monthly Notices of the Royal Astronomical Society, vol. 396, no. 3, pp. 1217-1221, 2009.

[99] P. F. Hopkins, L. Hernquist, T. J. Cox, B. Robertson, T. Di Matteo, and V. Springel, "The evolution in the faintend slope of the quasar luminosity function," Astrophysical Journal, vol. 639, no. 2, pp. 700-709, 2006.

[100] Q. Yu, Y. Lu, and G. Kauffmann, "Evolution of accretion disks around massive black holes: constraints from the demography of active galactic nuclei," Astrophysical Journal, vol. 634, no. 2, pp. 901-909, 2005.

[101] A. R. King and J. E. Pringle, "Fuelling active galactic nuclei," Monthly Notices of the Royal Astronomical Society, vol. 377, no. 1, pp. L25-L28, 2007.

[102] B. M. Peterson, L. Ferrarese, K. M. Gilbert et al., "Central masses and broad-line region sizes of active galactic nuclei. II. A homogeneous analysis of a large reverberation-mapping database," Astrophysical Journal Letters, vol. 613, no. 2, pp. 682-699, 2004.

[103] M. C. Bentz et al., "The lick AGN monitoring project: broadline region radii and black hole masses from reverberation mapping of H $\beta$," Astrophysical Journal, vol. 705, article 199, 2009.

[104] S. Kaspi, D. Maoz, H. Netzer, B. M. Peterson, M. Vestergaard, and B. T. Jannuzi, "The relationship between luminosity and broad-line region size in active galactic nuclei," Astrophysical Journal, vol. 629, no. 1, pp. 61-71, 2005.

[105] M. C. Bentz, B. M. Peterson, H. Netzer, R. W. Pogge, and M. Vestergaard, "The radius-luminosity relationship for active galactic nuclei: the effect of host-galaxy starlight on luminosity measurements. II. the full sample of reverberationmapped agns," Astrophysical Journal, vol. 697, no. 1, pp. 160181, 2009. 
[106] C. A. Onken, L. Ferrarese, D. Merritt et al., "Supermassive black holes in active galactic nuclei. II. Calibration of the black hole mass-velocity dispersion relationship for active galactic nuclei," Astrophysical Journal, vol. 615, no. 2, pp. 645651, 2004.

[107] J.-H. Woo, T. Treu, A. J. Barth et al., “The lick AGN monitoring project: the $\mathrm{MBH}-\sigma *$ relation for reverberation-mapped active galaxies," Astrophysical Journal Letters, vol. 716, no. 1, pp. 269-280, 2010.

[108] J. E. Greene and L. C. Ho, "Estimating black hole masses in active galaxies using the $\mathrm{H} \alpha$ emission line," Astrophysical Journal, vol. 630, no. 1, pp. 122-129, 2005.

[109] R. J. McLure and M. J. Jarvis, "Measuring the black hole masses of high-redshift quasars," Monthly Notices of the Royal Astronomical Society, vol. 337, no. 1, pp. 109-116, 2002.

[110] M. Vestergaard and P. S. Osmer, "Mass functions of the active black holes in distant quasars from the large bright quasar survey, the bright quasar survey, and the color-selected sample of the sdss fall equatorial stripe," Astrophysical Journal, vol. 699, no. 1, pp. 800-816, 2009.

[111] Y. Shen, G. T. Richards, M. A. Strauss et al., "A catalog of quasar properties from Sloan Digital Sky Survey Data Release 7," Astrophysical Journal Supplement Series, vol. 194, no. 2, article 45, 2011.

[112] J. A. Kollmeier, C. A. Unken, C. S. Kochanek et al., "Black hole masses and eddington ratios at $0.3<\mathrm{z}<4$," Astrophysical Journal, vol. 648, no. 1, pp. 128-139, 2006.

[113] Y. Shen, J. E. Greene, M. A. Strauss, G. T. Richards, and D. P. Schneider, "Biases in virial black hole masses: an SDSS perspective," Astrophysical Journal, vol. 680, no. 1, pp. 169$190,2008$.

[114] C. L. Steinhardt and M. Elvis, "The quasar mass-luminosity plane-III. Smaller errors on virial mass estimates," Monthly Notices of the Royal Astronomical Society, vol. 406, no. 1, pp. L1-L5, 2010.

[115] B. C. Kelly, M. Vestergaard, X. Fan, P. Hopkins, L. Hernquist, and A. Siemiginowska, "Constraints on black hole growth, quasar lifetimes, and Eddington ratio distributions from the SDSS broad-line quasar black hole mass function," Astrophysical Journal, vol. 719, no. 2, pp. 1315-1334, 2010.

[116] Y. Shen and B. C. Kelly, "The demographics of broad-line quasars in the mass-luminosity plane. I. testing FWHMbased virial black hole masses," Astrophysical Journal. In press.

[117] J.-M. Wang, Y. M. Chen, and F. Zhang, "Cosmological evolution of the duty cycle of quasars," Astrophysical Journal Letters, vol. 647, no. 1, pp. L17-L20, 2006.

[118] J. E. Greene and L. C. Ho, "The mass function of active black holes in the local universe," Astrophysical Journal, vol. 667, no. 1, pp. 131-148, 2007.

[119] M. Vestergaard, X. Fan, C. A. Tremonti, P. S. Osmer, and G. T. Richards, "Mass functions of the active black holes in distant quasars from the sloan digital sky survey data release 3," Astrophysical Journal, vol. 674, no. 1, pp. L1-L4, 2008.

[120] G. T. Richards, M. A. Strauss, X. Fan et al., "The sloan digital sky survey quasar survey: quasar luminosity function from data release 3," Astronomical Journal, vol. 131, no. 6, pp. 2766-2787, 2006.

[121] A. Schulze and L. Wisotzki, "Low redshift AGN in the Hamburg/ESO Survey: II. The active black hole mass function and the distribution function of Eddington ratios," Astronomy and Astrophysics, vol. 516, no. 15, article A87, 2010.

[122] M. Schmidt and R. F. Green, "Quasar evolution derived from the Palomar bright quasar survey and other complete quasar surveys," Astrophysical Journal, vol. 269, pp. 352-374, 1983.

[123] P. Natarajan and E. Treister, "Is there an upper limit to black hole masses?" Monthly Notices of the Royal Astronomical Society, vol. 393, no. 3, pp. 838-845, 2009.

[124] D. Sijacki, V. Springel, and M. G. Haehnelt, "Growing the first bright quasars in cosmological simulations of structure formation," Monthly Notices of the Royal Astronomical Society, vol. 400, no. 1, pp. 100-122, 2009.

[125] J. E. Greene and L. C. Ho, "Active galaxies and the study of black hole demographics," Publications of the Astronomical Society of the Pacific, vol. 121, no. 885, pp. 1167-1171, 2009.

[126] S. Collin, T. Kawaguchi, B. M. Peterson, and M. Vestergaard, "Systematic effects in measurement of black hole masses by emission-line reverberation of active galactic nuclei: eddington ratio and inclination," Astronomy and Astrophysics, vol. 456, no. 1, pp. 75-90, 2006.

[127] G. T. Richards, N. E. Kruczek, S. C. Gallagher et al., "Unification of luminous type 1 quasars through Civ emission," Astronomical Journal, vol. 141, no. 5, article 167, 2011.

[128] A. Marconi, D. J. Axon, R. Maiolino et al., "The effect of radiation pressure on virial black hole mass estimates and the case of narrow-line Seyfert 1 galaxies," Astrophysical Journal, vol. 678, no. 2, pp. 693-700, 2008.

[129] H. Netzer, "Radiation pressure force and black hole mass determination in low redshift type-I and type-II active galactic nuclei," The Astrophysical Journal, vol. 695, no. 1, article 793, 2009.

[130] A. Marconi, D. J. Axon, R. Maiolino et al., "On the observed distributions of black hole masses and eddington ratios from radiation pressure corrected virial indicators," The Astrophysical Journal Letters, vol. 698, no. 2, article L103, 2009.

[131] H. Netzer and P. Marziani, "The effect of radiation pressure on emission-line profiles and black hole mass determination in active galactic nuclei," Astrophysical Journal, vol. 724, no. 1, pp. 318-328, 2010.

[132] K. D. Denney, B. M. Peterson, M. Dietrich, M. Vestergaard, and M. C. Bentz, "Systematic uncertainties in measuring black hole masses from single epoch spectra," The Astrophysical Journal, vol. 692, article 246, 2009.

[133] R. I. Davies, J. Thomas, R. Genzel et al., "The star-forming torus and stellar dynamical black hole mass in the Seyfert 1 nucleus of NGC 3227," Astrophysical Journal, vol. 646, no. 2, pp. 754-773, 2006.

[134] E. K. S. Hicks and M. A. Malkan, "Circumnuclear gas in seyfert 1 galaxies: morphology, kinematics, and direct measurement of black hole masses," Astrophysical Journal Supplement Series, vol. 174, no. 1, pp. 31-73, 2008.

[135] C. A. Onken, M. Valluri, B. M. Peterson et al., "The black hole mass of NGC 4151: comparison of reverberation mapping and stellar dynamical measurements," Astrophysical Journal, vol. 670, no. 1, pp. 105-115, 2007.

[136] A. Siemiginowska and M. Elvis, "Deriving the quasar luminosity function from accretion-disk instabilities," Astrophysical Journal, vol. 482, no. 1, pp. L9-L12, 1997.

[137] E. Hatziminaoglou, A. Siemioinowska, and M. Elvis, "Accretion disk instabilities, cold dark matter models, and their role in quasar evolution," Astrophysical Journal, vol. 547, no. 1, pp. 90-98, 2001.

[138] A. Semicinowska, B. Czerny, and V. Kostyunin, "Evolution of an accretion disk in an active galactic nucleus," Astrophysical Journal, vol. 458, no. 2, pp. 491-507, 1996. 
[139] A. Franceschini, S. Vercellone, and A. C. Fabian, "Supermassive black holes in early-type galaxies: relationship with radio emission and constraints on the black hole mass function," Monthly Notices of the Royal Astronomical Society, vol. 297, no. 3, pp. 817-824, 1998.

[140] M. G. Haehnelt and M. J. Rees, "The formation of nuclei in newly formed galaxies and the evolution of the quasar population," Monthly Notices of the Royal Astronomical Society, vol. 263, no. 1, pp. 168-178, 1993.

[141] T. Hosokawa, "Constraining the lifetime of quasars with the present-day mass function of supermassive black holes," Astrophysical Journal, vol. 576, no. 1, pp. 75-80, 2002.

[142] A. Cavaliere and V. Vittorini, "Supermassive black holes in galactic nuclei," Astrophysical Journal, vol. 570, no. 1, pp. 114$118,2002$.

[143] M. Enoki, M. Nagashima, and N. Gouda, "Relations between galaxy formation and the environments of quasars," Publications of the Astronomical Society of Japan, vol. 55, no. 1, pp. 133-142, 2003.

[144] G. L. Granato, G. De Zotti, L. Silva, A. Bressan, and L. Danese, "A physical model for the coevolution of QSOs and their spheroidal hosts," Astrophysical Journal, vol. 600, no. 2, pp. 580-594, 2004.

[145] P. F. Hopkins, L. Hernquist, T. J. Cox, and D. Kerbs, "A cosmological framework for the co-evolution of quasars, supermassive black holes, and elliptical galaxies. i. galaxy mergers and quasar activity," Astrophysical Journal Supplement Series, vol. 175, no. 2, pp. 356-389, 2008.

[146] Y. Shen, "Supermassive black holes in the hierarchical universe: a general framework and observational tests," Astrophysical Journal Letters, vol. 704, no. 1, pp. 89-108, 2009.

[147] N. Fanidakis, C. M. Baugh, A. J. Benson et al., "Grand unification of AGN activity in the $\Lambda \mathrm{CDM}$ cosmology," Monthly Notices of the Royal Astronomical Society, vol. 410, no. 1, pp. 53-74, 2011.

[148] N. Fanidakis, Baugh C. M., A. J. Benson et al., "The evolution of active galactic nuclei across cosmic time: what is downsizing?" Monthly Notices of the Royal Astronomical Society. In press.

[149] F. Hoyle and R. A. Lyttleton, "The effect of interstellar matter on climatic variation," Mathematical Proceedings of the Cambridge Philosophical Society, vol. 35, no. 3, pp. 405-415, 1939.

[150] H. Bondi and F. Hoyle, "On the mechanism of accretion by stars," Monthly Notices of the Royal Astronomical Society, vol. 104, p. 273, 1944.

[151] H. Bondi, "On spherically symmetrical accretion," Monthly Notices of the Royal Astronomical Society, vol. 112, p. 195, 1952.

[152] M. Volonteri and M. C. Begelman, "Quasi-stars and the cosmic evolution of massive black holes," Monthly Notices of the Royal Astronomical Society, vol. 409, no. 3, pp. 1022-1032, 2010.

[153] L. Jiang, X. Fan, M. Vestergaard et al., "Gemini nearinfrared spectroscopy of luminous $\mathrm{z} \sim 6$ quasars: chemical abundances, black hole masses, and Mg II absorption," Astronomical Journal, vol. 134, no. 3, pp. 1150-1161, 2007.

[154] D. J. Mortlock, S. J. Warren, B. P. Venemans et al., "A luminous quasar at a redshift of $\mathrm{z}=7.085$," Nature, vol. 474, no. 7353, pp. 616-619, 2011.

[155] Z. Haiman and A. Loeb, "What is the highest plausible redshift of luminous quasars?" Astrophysical Journal Letters, vol. 552, no. 2, pp. 459-463, 2001.
[156] G. Lodato and P. Natarajan, "The mass function of highredshift seed black holes," Monthly Notices of the Royal Astronomical Society, vol. 377, no. 1, pp. L64-L68, 2007.

[157] G. Lodato and P. Natarajan, "Supermassive black hole formation during the assembly of pre-galactic discs," Monthly Notices of the Royal Astronomical Society, vol. 371, no. 4, pp. 1813-1823, 2006.

[158] M. C. Begelman, M. Volonteri, and M. J. Rees, "Formation of supermassive black holes by direct collapse in pre-galactic haloes," Monthly Notices of the Royal Astronomical Society, vol. 370, no. 1, pp. 289-298, 2006.

[159] M. C. Begelman, E. M. Rossi, and P. J. Armitage, "Quasi-stars: accreting black holes inside massive envelopes," Monthly Notices of the Royal Astronomical Society, vol. 387, no. 4, pp. 1649-1659, 2008.

[160] M. C. Begelman, "Evolution of supermassive stars as a pathway to black hole formation," Monthly Notices of the Royal Astronomical Society, vol. 402, no. 1, pp. 673-681, 2010.

[161] M. Volonteri, G. Lodato, and P. Natarajan, "The evolution of massive black hole seeds," Monthly Notices of the Royal Astronomical Society, vol. 383, no. 3, pp. 1079-1088, 2008.

[162] P. Natarajan and M. Volonteri, "The mass function of black holes at $1<\mathrm{z}<4.5$ : comparison of models with observations," Monthly Notices of the Royal Astronomical Society. In press.

[163] Z. Lippai, Z. Frei, and Z. Haiman, "On the occupation fraction of seed black holes in high-redshift dark matter halos," Astrophysical Journal Letters, vol. 701, no. 1, pp. 360$368,2009$.

[164] T. Tanaka and Z. Haiman, "The assembly of supermassive black holes at high redshifts," The Astrophysical Journal, vol. 696, p. 1798, 2009.

[165] E. Berti and M. Volonteri, "Cosmological black hole spin evolution by mergers and accretion," Astrophysical Journal, vol. 684, no. 2, pp. 822-828, 2008.

[166] K. Horne, B. M. Peterson, S. J. Collier, and H. Netzer, “Observational requirements for high-fidelity reverberation mapping," Publications of the Astronomical Society of the Pacific, vol. 116, no. 819, pp. 465-476, 2004.

[167] M. C. Bentz, K. Horne, A. J. Barth et al., "The lick agn monitoring project: velocity-delay maps from the maximumentropy method for Arp 151," Astrophysical Journal, vol. 720, no. 1, pp. L46-L51, 2010.

[168] A. Pancoast, B. J. Brewer, and T. Treu, "Geometric and dynamical models of reverberation mapping data," Astrophysical Journal Letters, vol. 730, no. 2, article 139, 2011.

[169] Y. Zu, C. S. Kochanek, and B. M. Peterson, "An alternative approach to measuring reverberation lags in active galactic nuclei," Astrophysical Journal Letters, vol. 735, no. 2, article 80, 2011.

[170] B. J. Brewer, T. Treu, A. Pancoast et al., "The mass of the black hole in Arp 151 from bayesian modeling of reverberation mapping data," Astrophysical Journal Letters, vol. 733, no. 2, article L33, 2011.

[171] A. Merloni, S. Heinz, and T. Di Matteo, "A fundamental plane of black hole activity: pushing forward the unification scheme," Astrophysics and Space Science, vol. 300, no. 1-3, pp. 45-53, 2005.

[172] E. Körding, H. Falcke, and S. Corbel, "Refining the fundamental plane of accreting black holes," Astronomy and Astrophysics, vol. 456, no. 2, pp. 439-450, 2006.

[173] K. Gültekin, E. M. Cackett, J. M. Miller, T. Di Matteo, S. Markoff, and D. O. Richstone, "The fundamental plane of accretion onto black holes with dynamical masses," Astrophysical Journal, vol. 706, no. 1, pp. 404-416, 2009. 
[174] R. M. Plotkin, S. Markoff, B. C. Kelly, E. Kö rding, and S. F. Anderson, "Using the Fundamental Plane of black hole activity to distinguish X-ray processes from weakly accreting black holes," Monthly Notices of the Royal Astronomical Society, vol. 419, no. 1, pp. 267-286, 2012.

[175] A. Laor and E. Behar, "On the origin of radio emission in radio-quiet quasars," Monthly Notices of the Royal Astronomical Society, vol. 390, no. 2, pp. 847-862, 2008.

[176] B. C. Kelly, J. Bechtold, and A. Siemiginowska, "Are the variations in quasar optical flux driven by thermal fluctuations?" Astrophysical Journal, vol. 698, no. 1, pp. 895-910, 2009.

[177] C. L. MacLeod, Z. Ivezić, C. S. Kochanek et al., "Modeling the time variability of SDSS Stripe 82 quasars as a damped random walk," Astrophysical Journal, vol. 721, no. 2, pp. 1014-1033, 2010.

[178] M. Brusa, F. Fiore, P. Santini et al., "Black hole growth and starburst activity at $\mathrm{z}=0.6-4$ in the Chandra Deep Field South," Astronomy and Astrophysics, vol. 507, no. 3, pp. 12771289, 2009.

[179] C. N. Cardamone, C. Megan Urry, K. Schawinski, E. Treister, G. Brammer, and E. Gawiser, "Dust-corrected colors reveal bimodality in the host-galaxy colors of active galactic nuclei at $\mathrm{z} \sim 1$," Astrophysical Journal, vol. 721, no. 1, pp. L38-L42, 2010.

[180] Y. Q. Xue, W. N. Brandt, B. Luo et al., "Color-magnitude relations of active and non-active galaxies in the Chandra Deep fields: high-redshift constraints and stellar-mass selection effects," Astrophysical Journal, vol. 720, no. 1, pp. 368$391,2010$.

[181] J. Aird, A. L. Coil, J. Moustakas et al., "PRIMUS: the dependence of AGN accretion on host stellar mass and color," The Astrophysical Journal. In press.

[182] D. M. Alexander, W. N. Brandt, I. Smail et al., "Weighing the black holes in Z 2 submillimeter-emitting galaxies hosting active galactic nuclei," Astronomical Journal, vol. 135, no. 5, pp. 1968-1981, 2008.

[183] J. E. Sarria, R. Maiolino, F. La Franca et al., "The MBH-Mstar relation of obscured AGNs at high redshift," Astronomy and Astrophysics, vol. 522, no. 1, article L3, 2010.

[184] A. Schulze and L. Wisotzki, "Selection effects in the black hole-bulge relation and its evolution," Astronomy and Astrophysics, vol. 535, article A87, 2011.

[185] M. Volonteri, "Gravitational recoil: signatures on the massive black hole population," Astrophysical Journal, vol. 663, no. 1, pp. L5-L8, 2007.

[186] L. Blecha and A. Loeb, "Effects of gravitational-wave recoil on the dynamics and growth of supermassive black holes," Monthly Notices of the Royal Astronomical Society, vol. 390, no. 4, pp. 1311-1325, 2008.

[187] D. Sijacki, V. Springel, and M. G. Haehnelt, "Gravitational recoils of supermassive black holes in hydrodynamical simulations of gas-rich galaxies," Monthly Notices of the Royal Astronomical Society, vol. 414, no. 4, pp. 3656-3670, 2011.

[188] L. Blecha, T. J. Cox, A. Loeb, and L. Hernquist, "Recoiling black holes in merging galaxies: relationship to active galactic nucleus lifetimes, starbursts and the $\mathrm{MBH}-\sigma *$ relation," Monthly Notices of the Royal Astronomical Society, vol. 412, no. 4, pp. 2154-2182, 2011.

[189] M. Volonteri, K. Gültekin, and M. Dotti, "Gravitational recoil: effects on massive black hole occupation fraction over cosmic time," Monthly Notices of the Royal Astronomical Society, vol. 404, no. 4, pp. 2143-2150, 2010.
[190] M. Volonteri, P. Natarajan, and K. Gültekin, "How important is the dark matter halo for black hole growth?" Astrophysical Journal, vol. 737, no. 2, article 50, 2011.

[191] L. Ciotti, J. P. Ostriker, and D. Proga, "Feedback from central black holes in elliptical galaxies. I. Models with either radiative or mechanical feedback but not both," Astrophysical Journal, vol. 699, no. 1, pp. 89-104, 2009.

[192] M. S. Shin, J. P. Ostriker, and L. Ciotti, "Feedback from central black holes in elliptical galaxies. II. Can purely mechanical energy feedback models work?" Astrophysical Journal, vol. 711, no. 1, pp. 268-283, 2010.

[193] L. Ciotti, J. P. Ostriker, and D. Proga, "Feedback from central black holes in elliptical galaxies. III. Models with both radiative and mechanical feedback," Astrophysical Journal, vol. 717, no. 2, pp. 708-723, 2010.

[194] R. Kurosawa, D. Proga, and K. Nagamine, "ON the feedback efficiency of active galactic nuclei," Astrophysical Journal, vol. 707, no. 1, pp. 823-832, 2009.

[195] J. P. Ostriker, E. Choi, L. Ciotti, G. S. Novak, and D. Proga, "Momentum driving: which physical processes dominate active galactic nucleus feedback?" Astrophysical Journal, vol. 722, no. 1, pp. 642-652, 2010.

[196] A. C. Fabian, J. S. Sanders, S. W. Allen et al., "A deep Chandra observation of the Perseus cluster: shocks and ripples," Monthly Notices of the Royal Astronomical Society, vol. 344, no. 3, pp. L43-L47, 2003.

[197] P. F. Hopkins and E. Quataert, "An analytic model of angular momentum transport by gravitational torques: from galaxies to massive black holes," Monthly Notices of the Royal Astronomical Society, vol. 415, no. 2, pp. 1027-1050, 2011.

[198] C. Power, S. Nayakshin, and A. King, "The accretion disc particle method for simulations of black hole feeding and feedback," Monthly Notices of the Royal Astronomical Society, vol. 412, no. 1, pp. 269-276, 2011. 

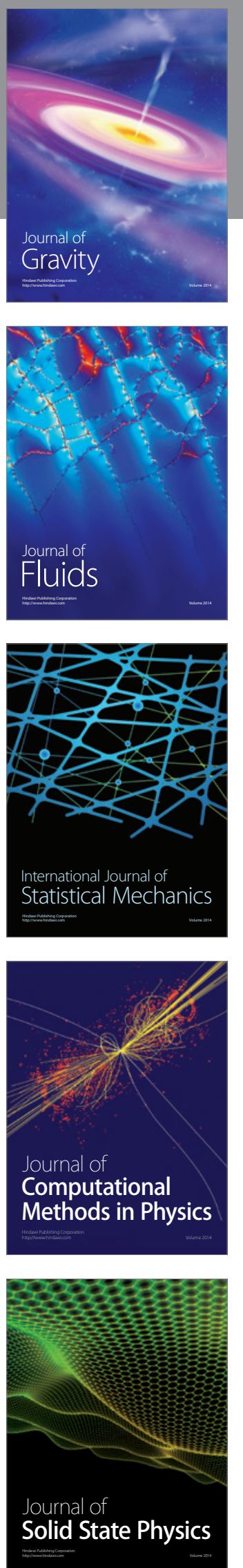

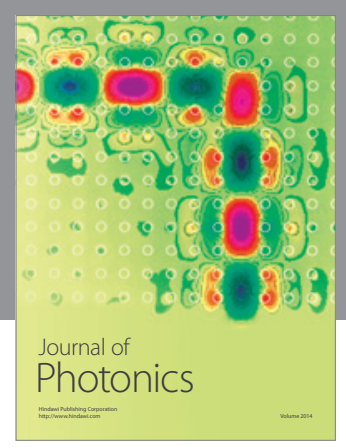

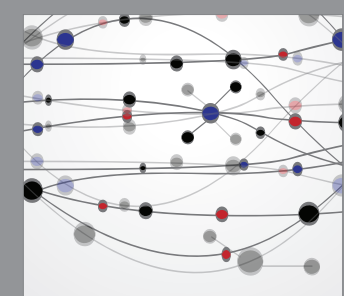

The Scientific World Journal
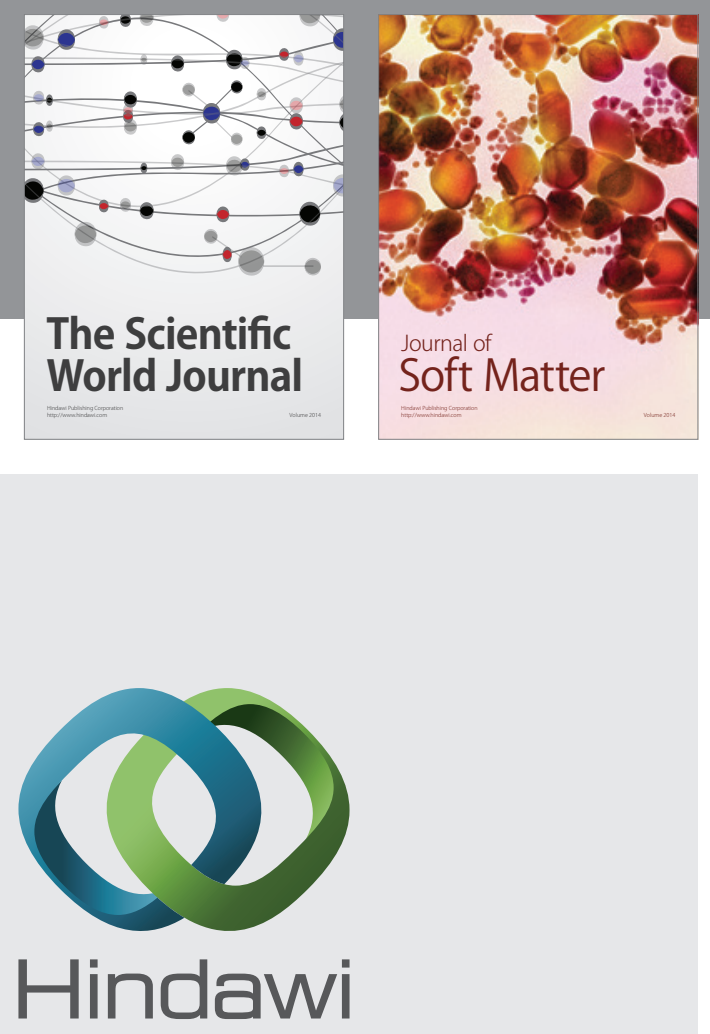

Submit your manuscripts at

http://www.hindawi.com
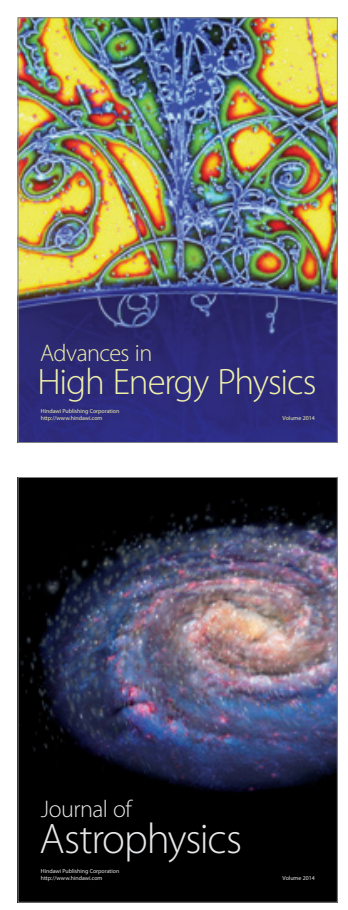
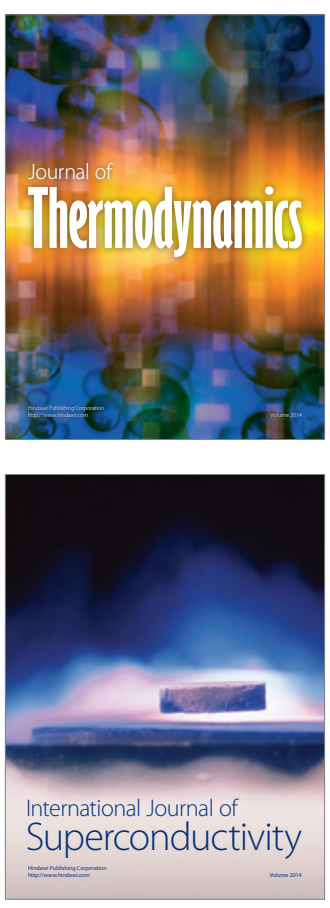
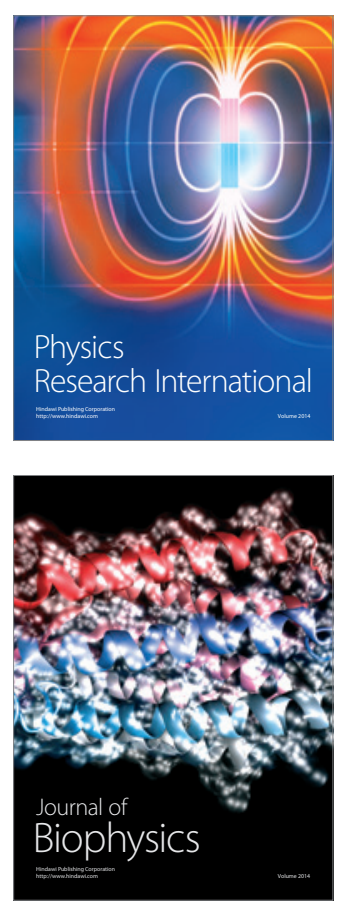
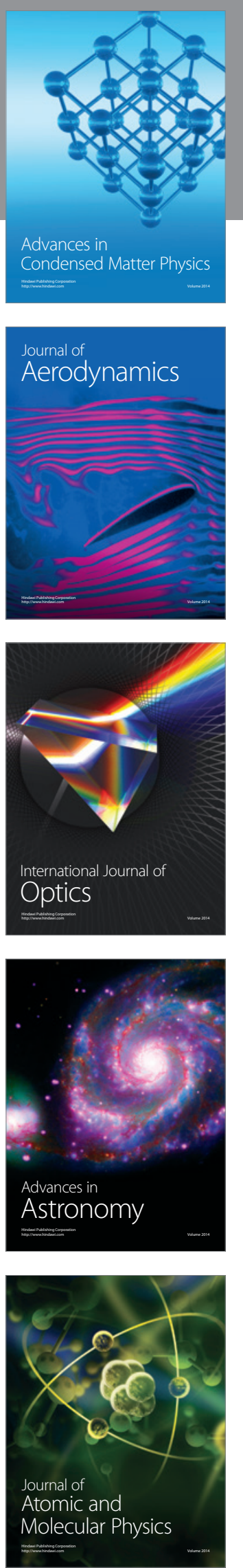